\title{
Dispersion interactions in room-temperature ionic liquids: Results from a non-empirical density functional
}

\author{
Jorge Kohanoff, ${ }^{1, \text { a) }}$ Carlos Pinilla, ${ }^{2,3}$ Tristan G. A. Youngs, ${ }^{1,4}$ Emilio Artacho, ${ }^{5}$ \\ and José M. Soler6 \\ ${ }^{1}$ Atomistic Simulation Centre, Queen's University Belfast, Belfast BT7 1NN, Northern Ireland \\ ${ }^{2}$ School of Chemistry, University of Bristol, Bristol BS2 ITS, United Kingdom \\ ${ }^{3}$ The Abdus Salam International Centre for Theoretical Physics (ICTP), Strada Costiera 11, \\ I-34151, Trieste, Italy \\ ${ }^{4}$ QUILL Research Centre, School of Chemistry and Chemical Engineering, Queens University Belfast, \\ Belfast BT7 INN, Northern Ireland \\ ${ }^{5}$ Department of Earth Sciences, University of Cambridge, Cambridge CB2 3EQ, United Kingdom \\ ${ }^{6}$ Departamento de Física de la Materia Condensada, Universidad Autónoma de Madrid, \\ ES-28049 Madrid, Spain
}

(Received 9 August 2011; accepted 28 September 2011; published online 20 October 2011)

\begin{abstract}
The role of dispersion or van de Waals (VDW) interactions in imidazolium-based room-temperature ionic liquids is studied within the framework of density functional theory, using a recently developed non-empirical functional [M. Dion, H. Rydberg, E. Schröder, D. C. Langreth, and B. I. Lundqvist, Phys. Rev. Lett. 92, 246401 (2004)], as efficiently implemented in the SIESTA code [G. RománPérez and J. M. Soler, Phys. Rev. Lett. 103, 096102 (2009)]. We present results for the equilibrium structure and lattice parameters of several crystalline phases, finding a general improvement with respect to both the local density (LDA) and the generalized gradient approximations (GGA). Similar to other systems characterized by VDW bonding, such as rare gas and benzene dimers as well as solid argon, equilibrium distances and volumes are consistently overestimated by $\approx 7 \%$, compared to $-11 \%$ within LDA and $11 \%$ within GGA. The intramolecular geometries are retained, while the intermolecular distances and orientations are significantly improved relative to LDA and GGA. The quality is superior to that achieved with tailor-made empirical VDW corrections ad hoc [M. G. Del Pópolo, C. Pinilla, and P. Ballone, J. Chem. Phys. 126, 144705 (2007)]. We also analyse the performance of an optimized version of this non-empirical functional, where the screening properties of the exchange have been tuned to reproduce high-level quantum chemical calculations [J. Klimes, D. Bowler, and A. Michaelides, J. Phys.: Condens. Matter 22, 074203 (2010)]. The results for solids are even better with volumes and geometries reproduced within $2 \%$ of experimental data. We provide some insight into the issue of polymorphism of $[\mathrm{bmim}][\mathrm{Cl}]$ crystals, and we present results for the geometry and energetics of $[\mathrm{bmim}][\mathrm{Tf}]$ and $[\mathrm{mmim}][\mathrm{Cl}]$ neutral and charged clusters, which validate the use of empirical force fields. @ 2011 American Institute of Physics. [doi:10.1063/1.3652897]
\end{abstract}

\section{INTRODUCTION}

In the past decade room-temperature ionic liquids (RTILs) have been extensively explored as a new class of environmentally friendly medium for many applications such as chemical synthesis and electrochemical cells, to name but a few. ${ }^{1}$ These salts are generally composed of a large cation, e.g., the aromatic dialkyl-imidazolium or the aliphatic alkylammonium families, together with a variety of anions ranging from elemental halogens $\left(\mathrm{Cl}^{-}\right)$to more complex entities such as hexafluorophosphate $\left(\mathrm{PF}_{6}^{-}\right)$, triflate $(\mathrm{Tf})$, and bistriflamide $\left(\mathrm{NTf}_{2}\right)$. Common to most members of this combinatorially large family is a low melting point (around or slightly above room temperature, as opposed to inorganic molten salts which possess melting points in excess of $1000 \mathrm{~K}$ ) together with a clear ionic character. Naturally, electrostatic interactions are a major source of cohesion. However, the bulky characteristic of the cations (and in some cases also the anions) marks

\footnotetext{
${ }^{a)}$ Electonic mail: j.kohanoff@qub.ac.uk.
}

a significant difference with respect to traditional inorganic salts such as $\mathrm{NaCl}$. In imidazolium-based salts, the cation's charge is concentrated in the aromatic ring, while the side alkyl chains exhibit mostly a non-polar, closed-shell character. Given that these occupy a significant volume fraction in condensed phases (either liquid, crystalline, or aggregates), there is scope for a significant contribution to cohesion arising from dispersion, van der Waals (VDW)-type interactions between the side chains of the cations.

Much has been learnt about RTILs through computer simulation. $^{2}$ The main tool has been molecular dynamics where the interactions between atoms are described via empirical force fields. The main reason behind this choice is that the dynamics of RTILs is generally very slow, almost glassy. Therefore, to achieve the time scale required to study certain important dynamical phenomena such as diffusion, it is necessary to run simulations of the order of several nanoseconds. Irrespective of the advances in first-principles molecular dynamics methods and the increase in computer power, this time scale is still beyond their reach. Hence, the best available 
strategy is to to develop high-quality classical force fields based on quantum-mechanical calculations. Most of the force fields available for RTILs have been obtained by fitting the model parameters to high-level quantum-chemical gas-phase calculations. ${ }^{3}$ This approach proved very effective, especially when care was taken in identifying and reproducing the most relevant features of the potential energy surface. In the case of dialkyl-imidazolium cations, the torsional potential of the alkyl chains was identified as one of the key ingredients. ${ }^{3}$

Nevertheless, this methodology remains based on gasphase calculations, and the peculiarities of the condensed phase are generally not included. ${ }^{4}$ Inter-molecular interactions in these models are represented by electrostatic interactions between partial charges at the atomic sites and repulsion-dispersion terms of the Lennard-Jones or Buckingham type to represent closed-shell effects. Partial charges are obtained by fitting the electrostatic potential in the gas phase, and thus miss possible charge redistribution and polarization effects occurring in the condensed phase. Repulsion-dispersion interactions, though, being shortranged, are not significantly affected by the environment. In order to address this issue, in the past some of us proposed a methodology to develop classical force fields based on first-principles, Car-Parrinello-like molecular dynamics simulations in the liquid phase. ${ }^{5}$ This method uses the forcematching strategy combined with simplex minimization and simulated annealing procedures and projections to separate the various classes of parameters in the model. ${ }^{6}$ This procedure was successfully applied to the RTIL [mmim][Cl], and a new set of partial charges and Lennard-Jones parameters was determined to reproduce the forces in the liquid phase. When comparing these results to experimental neutron scattering data, ${ }^{7}$ it turned out that the agreement in pair correlation functions could be improved by increasing the Lennard-Jones radii $(\sigma)$. The reason for this feature was readily traced back to the poor description of dispersion interactions in standard density-functional approaches, on which the first-principles liquid phase simulations were based. ${ }^{5}$ In particular, these simulations used the generalized gradient approximation to exchange and correlation, as proposed by Perdew, Burke and Ernzerhof (GGA-PBE). ${ }^{8}$ Another unsatisfactory feature of this model, most likely due to the fitting strategy that did not include information about lattice constants, was that the density of the liquid at room temperature was overestimated. It became clear then that, in order to obtain useful dispersion model parameters from condensed phase simulations, it was required a step forward in the description of exchange and correlation, going beyond the GGA and including van der Waals interactions.

The extent of the limitations of both, the LDA and the GGA, was thoroughly studied by Del Pópolo et al., ${ }^{9}$ who analyzed the structural properties of a family of imidazoliumbased RTILs for which the crystalline structures had been experimentally determined via x-ray diffraction. These results showed that, as expected, the equilibrium volume predicted by either the LDA or the PBE-GGA is affected by significant errors $(\approx 10 \%$, too small for LDA and too large for GGA). The intramolecular geometry was described reasonably well by both approximations while the description of the intermolec- ular geometry differed between the two approximations, both deviating significantly from the experimental data. Interestingly, it was found that the GGA-PBE intramolecular geometry obtained at the experimental unit cell parameters reproduced quite well the experimental data. An improvement was achieved by supplementing the PBE-GGA functional with a simple pair-potential model for the dispersion interactions, as previously done by several authors for van der Waals solids and liquids. ${ }^{10}$ However, the quantitative accuracy of these methods depends crucially on the parameters introduced in the correction, and a satisfactory agreement with experiment appears to require a large number of parameters and a careful and lengthy calibration. Moreover, the transferability of such parameters is not guaranteed even within a family of related compounds such as the imidazolium salts.

In recent years, a significant amount of work has been devoted to the development of correlation functionals capable of describing dispersion interactions. One such promising approach ${ }^{11}$ was very recently implemented in an efficient way ${ }^{12}$ into the SIESTA code for electronic structure calculations. ${ }^{13}$ In this paper, we analyze the performance of this non-empirical van der Waals density functional for the structural and energetic properties of a family of imidazoliumbased RTILs as done in Ref. 9, and we also address this issue in the case of neutral and charged aggregates, in order to support recent calculations aiming at understanding the properties of the vapour phase of RTILs. ${ }^{14}$ In addition, we assess the performance of a tuned-up version of this functional. ${ }^{15}$

\section{THEORY AND COMPUTATIONAL ASPECTS}

\section{A. The van der Waals functional}

The van der Waals attraction between closed-shell fragments is a well-known non-local correlation phenomenon. It originates from the dynamic coupling between charge density fluctuations in two spatially separated regions. It is therefore not surprising that local and semi-local approximations to correlation such as the LDA and the GGA fail to account for it. The best known aspect of the VDW interaction is its $R^{-6}$ attractive behaviour at long distances $(R)$ between fragments. However, this is not the whole story. When such fragments approach each other to the point where their electronic densities begin to overlap, this distance dependence is modified in an, a priori, unknown form. Semiempirical approaches take this into account by adding a density-independent term of the general form

$$
E_{\mathrm{VDW}}(\mathbf{R})=-\sum_{i j} f^{i j}\left(R_{i j}\right) \frac{C_{6}^{i j}\left(R_{i j}\right)}{R_{i j}^{6}},
$$

to the local or semi-local exchange-correlation functional. Here, the sum runs over all pairs of atoms in the system. The $C_{6}$ coefficients depend on the chemical identity of the atoms and are well-known and available in the literature for most elements, ${ }^{16}$ and the function $f$ eliminates smoothly the $1 / R^{6}$ divergence for $R \rightarrow 0$.

While this is an attractive alternative, especially due to its simplicity, ease of implementation, and low computational cost (it is simply a pair potential), it requires a non-trivial 
calibration step to determine its parameters. If the number of parameters is kept to a minimum, e.g., by choosing a single function $f$ for all pairs of atoms, the quality of this approximation rapidly finds its limitations, especially in RTILs that involve a variety of atomic species in various bonding environments. On the other hand, letting $f^{i j}$ depend on the chemical identity of the atoms makes the fitting process quite involved and also compromises the transferability of the potential. ${ }^{9}$

The exact expression for the VDW interaction is known. It is given by the integral of the coupling of density fluctuations at all frequencies. The problem is that such densitydensity response function requires the knowledge of the electron pair correlation function, which is generally unknown for an inhomogeneous system. The form of a non-local interaction from a density functional theory (DFT) point of view is

$$
E_{C}^{n l}[\rho]=\frac{1}{2} \iint \rho(\mathbf{r}) \Phi\left(\mathbf{r}, \mathbf{r}^{\prime}\right) \rho\left(\mathbf{r}^{\prime}\right) d \mathbf{r} d \mathbf{r}^{\prime} .
$$

This expression, which includes the VDW interaction, can be used to supplement the usual semi-local functional as

$$
E_{\mathrm{XC}}[\rho]=E_{X}^{\mathrm{GGA}}[\rho]+E_{C}^{\mathrm{LDA}}[\rho]+E_{C}^{n l}[\rho],
$$

where exchange is still treated at a semi-local level (GGA), while the semi-local component of the correlation is included in the new, fully non-local functional (2). The choice of the exchange GGA requires some care. It has been argued that the exchange functional must be such that the underlying exchange potential is always repulsive. Of the numerous semi-local functional proposed along the years, the socalled revPBE flavor ${ }^{17}$ fulfils approximately this requirement, as noted by Dion et al. ${ }^{11}$

Many attempts have been made at developing such a nonlocal correlation functional along the years, ${ }^{18}$ sometimes in the form of a weighted density approximation. A particularly successful version, which fulfils one of the fundamental symmetries of the functional, namely against the exchange of $\mathbf{r}$ and $\mathbf{r}^{\prime}$, has been recently developed by Dion et al. ${ }^{11}$ In this attempt, the kernel $\Phi\left(q, q^{\prime},\left|\mathbf{r}-\mathbf{r}^{\prime}\right|\right)$ is expressed in terms of a local momentum variable that depends on the density and its gradient,

$$
q[\rho, \nabla \rho]=k_{F}\left[1+\frac{\epsilon_{C}^{\mathrm{LDA}}[\rho]}{\epsilon_{X}^{\mathrm{LDA}}[\rho]}+\frac{0.8491}{9}\left(\frac{\nabla \rho}{2 \rho k_{F}}\right)^{2}\right],
$$

where $k_{F}=\left(3 \pi^{2} \rho\right)^{1 / 3}$ is the Fermi momentum and $\epsilon_{X}^{\mathrm{LDA}}, \epsilon_{C}^{\mathrm{LDA}}$ are the exchange and correlation energy densities of the homogeneous electron gas, respectively.

The non-local correlation kernel $\Phi\left(q, q^{\prime},\left|\mathbf{r}-\mathbf{r}^{\prime}\right|\right)$ proposed in Ref. 11 fulfils a number of conditions, such as integrating to zero for a uniform density, so that $E_{C}^{n l} \rightarrow 0$ and the (exact) LDA is recovered. Also, at large separations $\Phi$ $\rightarrow-C /\left|\mathbf{r}-\mathbf{r}^{\prime}\right|^{6}$ as required to reproduce the long-range $R^{-6}$ attraction between closed-shell fragments. Details of how $\Phi$ is constructed can be found in the original publications. ${ }^{11}$ Here, it is sufficient to say that this constitutes a general-purpose, seamless functional that, from now on, will be referred to as DRSLL.

This new functional was initially applied to $\mathrm{Ar}$ and $\mathrm{Kr}$ dimers, where binding is purely due to dispersion forces, and showed a dramatic improvement with respect to the GGA (see Fig. 2 in Ref. 11). While the GGA does not bind the dimer, DRSLL produces bond lengths that are only $\approx 5 \%$ too long and binding energies $\approx 50 \%$ too large in comparison to experimental data. This represented a major triumph compared to the performance of the GGA. Similar results were observed for the benzene dimer, where the overestimation of the binding energy turned out to be smaller $(\approx 30 \%)$. Moreover, DRSLL produced a binding energy curve in close agreement with high-level quantum chemistry methods- $\operatorname{CCSD}(\mathrm{T})$, and significantly better than MP2, which is the lowest level that includes dynamical correlations. The discrepancy in binding energies was shown to decrease in systems with increasingly extended valence electron states such as polycyclic aromatic hydrocarbon dimers, ${ }^{19}$ reducing to only $16 \%$ for naphthalene. For benzene interacting with water and some aliphatic molecules, the improvement on bond lengths was less dramatic. In fact, since in these systems there is some degree of covalency, the GGA does a respectable job for the geometry, but binding energies are still quite poor. These latter are dramatically improved by the DRSLL functional, although now the behaviour is more erratic, in some cases underestimating and in others overestimating, but always to within $10 \%$ of the CCSD(T) results. ${ }^{21}$ For molecular crystals such as polyethylene $^{22}$ and crystals of carbon nanotubes, it produced lattice parameters in very good agreement with experimental data. An important additional aspect is that the DRSLL functional does not compromise the accuracy of local and semilocal functionals for covalently bonded systems such as bulk $\mathrm{Si}^{23}$ These are only a few examples of an increasing number of applications-for a comprehensive review, see Ref. 19, and for a very recent application to metallic, ionic, and semiconducting solids, see Ref. 20. To the best of our knowledge, no DRSLL studies of gas or condensed phase ionic organic systems have been published to the date.

Very recently, some of the limitations of the DRSLL functional were discussed by Klimes et al., ${ }^{15}$ who proposed a modification of the parameters involved in the revPBE exchange functional while retaining the functional form of the enhancement factor

$$
F_{X}(s)=1+\kappa-\frac{\kappa}{1+\mu s^{2} / \kappa}
$$

where $s=|\nabla \rho| /\left(2 k_{F} \rho\right)$ and $k_{F}$ is the Fermi momentum. The only difference between $\mathrm{PBE}$ and revPBE is that the parameter $\kappa$, which originally was assigned the value $\kappa=0.804$, is modified to $\kappa=1.245$ in revPBE. Both functionals use $\mu=0.21951$, which provides a good description of atomization energies. ${ }^{24}$ By analyzing the behaviour of the exchange functional for the S22 database of molecular systems as a function of parameters $\kappa$ and $\mu$, these authors found that an optimized version with $\kappa=1$ and $\mu=0.23$ reproduces to an excellent extent results obtained by high-level - CCSD(T) - quantum chemistry calculations. The value of the coefficient $\mu$ has been subject of recent investigations. ${ }^{24}$ It has been argued that for solids with slowly varying densities, the choice of $\mu=0.1235$ (PBEsol functional), which recovers the correct gradient expansion of the enhancement factor for small gradients, is a better choice than the original PBE value, 
resulting in much improved lattice constants. This, however, does not apply to the present case of molecular ionic crystals, where bonding is mainly due to electrostatic and dispersion forces. The electronic density is localized in the ions and decays rapidly to very small values in the interstitial regions. Therefore, we chose the original PBE value $\mu=0.21951$ in order to retain the good energetic properties of PBE exchange. Parameter $\kappa$, on the other hand, must be such that the LiebOxford bound, stating that the total exchange energy must be lower than -1.679 a.u., is satisfied. ${ }^{25}$ Values lower than 0.804 guarantee that this inequality is verified locally, but this is not a necessary condition. Therefore, larger values may still be allowed, and thus we decided to assess the quality of the DRSLL functional where the revPBE exchange was modified so that $\kappa=1$. We shall call this functional " $\kappa=1$."

\section{B. Implementation}

Non-local correlation energy expressions have existed for a long time in the literature, e.g., as in the weighted density approximation. $^{26}$ The reason why they have not become of popular use is because of their excessive computational cost. The origin of this is the double integral in Eq. (2). Even if the distance $\left|\mathbf{r}-\mathbf{r}^{\prime}\right|$ is truncated beyond a reasonable cutoff $r_{c}$ $\approx 15 \AA$, and if order- $N$ techniques are used for systems of size larger than $2 r_{c}$, the direct calculation of the double integral remains a formidable task, at least ten times more expensive than the LDA or the GGA.

This limitation has been recently circumvented by the introduction, by one of us, of an efficient factorization technique. ${ }^{12}$ In this methodology, the non-local correlation kernel is expanded in a basis set of functions $p_{\alpha}(q)$

$$
\Phi\left(q, q^{\prime},\left|\mathbf{r}-\mathbf{r}^{\prime}\right|\right)=\sum_{\alpha, \beta} p_{\alpha}(q) p_{\beta}\left(q^{\prime}\right) \Phi_{\alpha \beta}\left(\left|\mathbf{r}-\mathbf{r}^{\prime}\right|\right)
$$

in such a way that the correlation energy can be computed as a sum of simple convolutions

$$
E_{C}^{n l} \approx \frac{1}{2} \sum_{\alpha, \beta} \iint \theta_{\alpha}(\mathbf{r}) \theta_{\beta}\left(\mathbf{r}^{\prime}\right) \Phi_{\alpha \beta}\left(\left|\mathbf{r}-\mathbf{r}^{\prime}\right|\right) d \mathbf{r} d \mathbf{r}^{\prime}
$$

where $\theta_{\alpha}(\mathbf{r})=\rho(\mathbf{r}) p_{\alpha}(q[\rho(\mathbf{r}, \nabla \rho(\mathbf{r})])$. The basis functions $p_{\alpha}(q)$ are chosen as cubic splines defined between consecutive grid points on a logarithmic mesh. A careful convergence analysis showed that 20 grid points, supplemented with an appropriate smoothing for $q \rightarrow 0$, are sufficient. Then, the convolutions can be carried out using FFT techniques. The number of convolutions is the of the order of 20 , and it is virtually independent of the system size. It does not require super cells for periodic systems, and there is no cutoff in the range of the interactions. ${ }^{12}$ Therefore, the efficiency of the algorithm increases with the size of the system, and for a sufficiently large number of atoms $(\approx 100)$, the overhead with respect to an LDA or a GGA calculation amounts only to about $20 \%$. For molecules or clusters, the overhead seems to be larger because the contribution of the exchange-correlation term represents a larger fraction of the calculation.

\section{Details of the calculations}

The calculations were carried out using the SIESTA code, ${ }^{13}$ which is based on pseudopotentials and a localized basis set of pseudoatomic orbitals. Pseudopotentials of the Troullier-Martins type ${ }^{27}$ have been generated using the same exchange-correlation approximations to be used for the crystals and clusters, i.e., LDA, PBE, DRSLL, and " $\kappa=1$." For the reference atomic configuration and pseudopotential core radii, we have used the same as in Ref. 9. We used a doublezeta plus polarization basis set (DZP). Careful testing of the localization radii of the basis functions used in Ref. 9 showed that the original values produced large basis set superposition errors (BSSE), and thus an overestimation of the cohesive energies. In order to improve our description, we have generated new DZP basis sets according to the procedure described in Ref., 30 which corrects on known deficiencies of the ones generated by the energy shift criterion. These deficiencies are, namely, (i) the external radii for anions are too short (and unnecessarily long for cations), (ii) the secondzeta matching radii are too long, thus giving a too limited radial flexibility, and (iii) the polarization orbitals generated by an external electric field ${ }^{28}$ are slightly too wide. In the new procedure, the latter are substituted by explicit, softly confined orbitals, ${ }^{29}$ and all of the relevant parameters are obtained variationally, ${ }^{29,30}$ resulting in the data shown in Table I. The soft-confinement potential parameters reported correspond to the following expression:

$$
V(r)=V_{0} \frac{e^{-\left(r_{c}-r_{i}\right) /\left(r-r_{i}\right)}}{r_{c}-r} .
$$

TABLE I. Basis set parameters as given in Eq. (8). The two numbers in third

\begin{tabular}{|c|c|c|c|c|}
\hline Species & Orbital & $r_{c}$ & $r_{i}$ & $V_{0}$ \\
\hline \multirow[t]{2}{*}{$\mathrm{H}$} & $s$ & $7.0,2.92$ & 6.0 & 50. \\
\hline & $p$ & 6.0 & 0.0 & 1000. \\
\hline \multirow[t]{3}{*}{$\mathrm{C}$} & $s$ & $7.0,3.2$ & 6.0 & 50. \\
\hline & $p$ & $7.0,3.3$ & 6.0 & 50. \\
\hline & $d$ & 6.0 & 3.8 & 100 \\
\hline \multirow[t]{3}{*}{$\mathrm{N}$} & $s$ & $7.0,2.8$ & 5.0 & 50. \\
\hline & $p$ & $7.0,2.8$ & 6.0 & 10. \\
\hline & $d$ & 6.0 & 1.0 & 50. \\
\hline \multirow[t]{3}{*}{$\mathrm{O}$} & $s$ & $7.0,2.4$ & 5.0 & 50. \\
\hline & $p$ & $7.0,2.2$ & 6.0 & 10. \\
\hline & $d$ & 6.0 & 0.0 & 50. \\
\hline \multirow[t]{3}{*}{$\mathrm{F}$} & $s$ & $7.0,2.2$ & 5.5 & 50. \\
\hline & $p$ & $7.0,2.2$ & 6.0 & 10. \\
\hline & $d$ & 6.0 & 0.0 & 50. \\
\hline \multirow[t]{3}{*}{$\mathrm{S}$} & $s$ & $6.5,4.4$ & 4.0 & 15. \\
\hline & $p$ & $6.5,4.0$ & 5.0 & 15. \\
\hline & $d$ & 5.0 & 2.0 & 100 \\
\hline \multirow[t]{3}{*}{$\mathrm{P}$} & $s$ & $6.5,5.0$ & 5.8 & 40. \\
\hline & $p$ & $6.5,4.6$ & 5.8 & 50. \\
\hline & $d$ & 5.0 & 2.0 & 100. \\
\hline \multirow[t]{3}{*}{$\mathrm{Cl}$} & $s$ & $6.5,3.7$ & 4.0 & 15. \\
\hline & $p$ & $6.5,3.7$ & 5.0 & 15. \\
\hline & $d$ & 6.5 & 6.0 & 100 \\
\hline
\end{tabular}
column correspond to the two $\zeta$ functions in the DZP basis. Radii in Bohr and $V_{0}$ in Ry. 
The electronic Brillouin zone of the crystals was sampled using the scheme of Moreno and Soler, with a real-space cutoff of $10 \AA .{ }^{31}$ This resulted in a number of $\mathbf{k}$-points that varied between 3 and 14, according to the size of the supercell. Previous convergence tests showed that this choice is sufficient, as the energy bands for the present wide bandgap molecular crystals are quite flat. ${ }^{9}$ Full optimization of the crystal lattice parameters and atomic positions was carried out using a combined conjugate gradients strategy as implemented in the SIESTA code. The optimizations were started from the previously obtained LDA and PBE structures ${ }^{9}$ for verification. For the DRSLL and " $\kappa=1$ " functionals, the optimizations were started from the PBE and DRSLL optimized structures, respectively.

At variance with traditional inorganic salts, we define the cohesive energy of the present ionic crystals with respect to the ground state energy of the neutral ion-pair, i.e., the cohesive energies reported below represent the energy required to decompose the crystal into gas-phase neutral pairs made of an anion and a cation. This choice highlights the role of intermolecular interactions, which are dominated by the van der Waals contribution. If we were to present the cohesive energies relative to the individual ions, rather than the ion pair, we would be including a substantial electrostatic contribution that would mask the dispersion component. The ion-pair ground state energies $\left(E_{\text {pair }}\right)$ were obtained by optimizing the atomic coordinates in a cubic supercell of $25 \AA$ side. Although less important than before, the present basis set still suffers from a BSSE of the order of $0.2 \mathrm{eV}$, and slightly larger for systems involving the $\mathrm{Cl}^{-}$anion. Since our cohesive energies are of the order of $1-2 \mathrm{eV}$, it is important to apply the counterpoise correction, and we have done so for all the energies reported in this paper.

Our optimizations provide the crystal structure at $T$ $=0 \mathrm{~K}$ and in the absence of zero-point motion. Experiments, however, have been carried out at finite temperature, and thus comprise those components. Including such effects in our first-principles calculations would be an extremely intensive computational task. An affordable alternative was proposed in Ref. 9, which consists of subtracting these contributions from the experimental data using accurate classical force fields, ${ }^{3}$ which reproduce reasonably well the vibrational spectrum of these systems. The free energy is calculated assuming the validity of the quasi-harmonic approximation, ${ }^{32}$ and the $T=0 \mathrm{~K}$ structure is obtained by extrapolation. We have thus used for comparison the extrapolated structural parameters obtained in Ref. 9.

For clusters an energetically important feature is the ionization energy defined as $E_{I}=E_{\text {pair }}-\left(E_{c a t}+E_{a n}\right)$, where $E_{c a t}$ and $E_{a n}$ are the energies of the cation and anion, respectively, and $E_{\text {pair }}$ was defined above. $E_{I}$ is important for the composition of the gas phase ${ }^{14}$ because it determines the relative population of charged species in the vapour. To compute the energy of the charged species, we carried out the SIESTA calculations in cubic supercells of $25 \AA$ side, with a compensating uniform background of the opposite charge to avoid the divergence of the electrostatic energy in periodic systems. The energies of the isolated ions were obtained by subtracting the Madelung correction as proposed by Leslie and Gillan. ${ }^{33}$

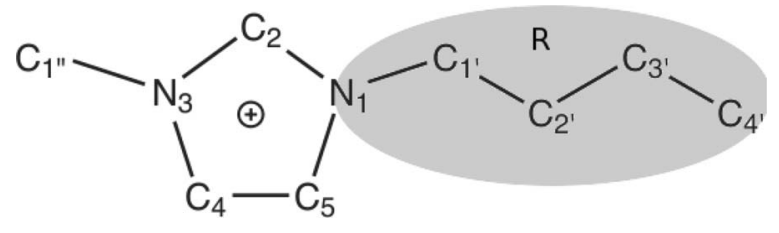

FIG. 1. Schematic picture of the 1-alkyl-3-methyl imidazolium cation. In this case the side group, indicated by a shaded area, is a butyl chain, thus leading to the bmim cation.

This correction assumes the form $\Delta E=-\alpha q^{2} / 2 \varepsilon_{0} L$, with $\alpha$ the Madelung constant ( $\alpha=5.6746$ for a simple cubic cell), $q$ the net charge in the supercell, $\varepsilon_{0}$ the dielectric constant of the vacuum, and $L$ the side length of the supercell. ${ }^{33} \mathrm{~A}$ second important quantity is the binding energy of neutral pairs, which is defined analogously to the cohesive energy of the crystals, i.e., without involving charged species. Here we only consider the binding of two ion-pairs to make a dimer, and thus the binding energy is $E_{\text {bind }}=E_{\text {dimer }}-2 E_{\text {pair }}$.

\section{RESULTS}

\section{A. Structure and energetics of 1-alkyl-3-methyl imidazolium room-temperature ionic liquid crystals}

Following Ref. 9, we have optimized the structure of a sequence of [R-mim] $\left[\mathrm{PF}_{6}\right]$ and [R-mim] [Cl] RTILs for which there is crystallographic data available. Here, $\mathrm{R}$ is the notation for a set of side groups of increasing size: methyl [mmim], ethyl [emim], butyl [bmim], and dodecyl [ddmim], involving 1, 2, 4, and 12 carbon atoms in the side chain (see Fig. 1). This choice of compounds is useful to assess the quality of the functional as a function of the magnitude of the VDW interaction relative to electrostatics. Salts involving cations with a larger hydrocarbon tail such as $\left[\mathrm{ddmim}^{2}\left[\mathrm{PF}_{6}\right]\right.$ should exhibit the largest deviations with respect to local and semi-local functionals, as this is the main source for the dispersion interaction. In Fig. 2, we show the crystal structures of two members of this family: $\left[\mathrm{mmim}^{2}\left[\mathrm{PF}_{6}\right]\right.$ exhibits the shortest side chain, and $[\mathrm{ddmim}]\left[\mathrm{PF}_{6}\right]$ exhibits the longest one. We also analyse the role of electrostatic interactions by studying two different anions, a stronger $\mathrm{Cl}^{-}$and a weaker $\mathrm{PF}_{6}^{-}$. In the case of [bmim] [Cl], we studied two reported crystalline forms: orthorhombic [bmim $][\mathrm{Cl}]-\mathrm{o}$ and monoclinic $[\mathrm{bmim}][\mathrm{Cl}]-\mathrm{m}$.

In Table II, we present the calculated equilibrium volumes of all the studied crystals at $T=0 \mathrm{~K}$. The experimental zero-temperature volumes have been obtained by extrapolation from finite temperature data ${ }^{34,35}$ by Del Pópolo et al. ${ }^{9}$ The volumes obtained using the van der Waals functional (DRSLL) are clearly closer to experiment than LDA and GGA-PBE volumes. In line with the general trends observed for closed-shell systems, the DRSLL functional still overestimates volumes by $7 \%-8 \%$. Even better agreement $(2 \%-4 \%)$ is obtained if the $\kappa=1$ functional is used. Very similar conclusions were extracted in a recent study of lattice constants for a variety of covalent and ionic solids, namely that the original functional of Dion et al. overestimates volumes, while the tuned-up versions produce results in better agreement with experiment. ${ }^{20}$ It can be seen, however, that 

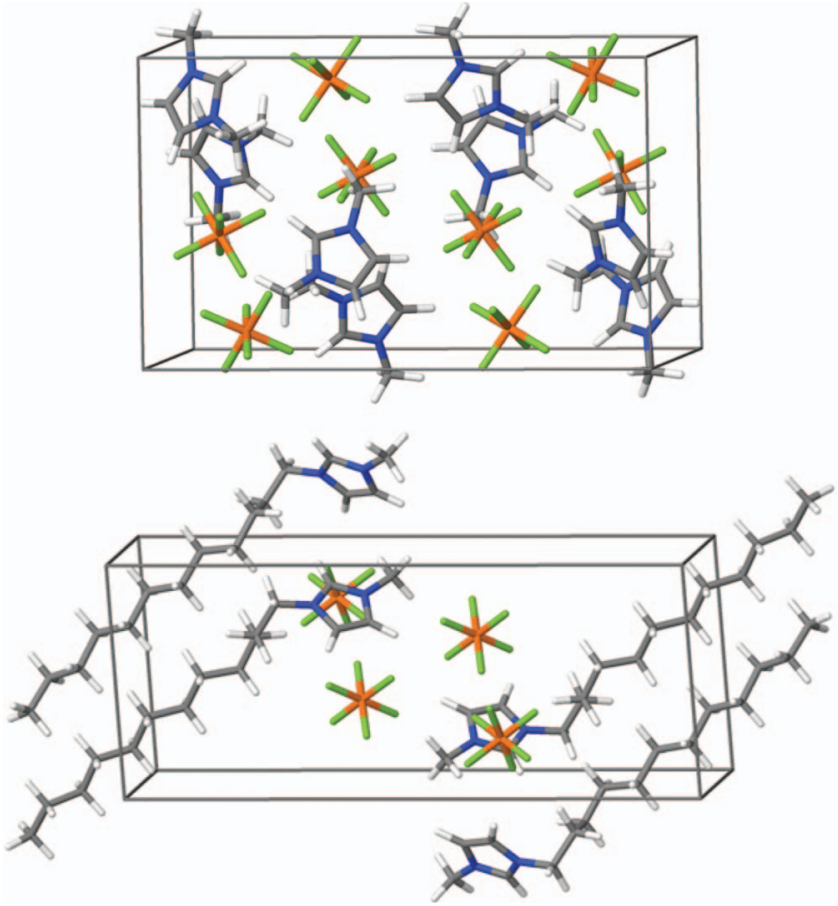

FIG. 2. Crystal structures of the hexafluorophosphate solids $[\mathrm{mmim}]\left[\mathrm{PF}_{6}\right]$ (top) and [ddmim $]\left[\mathrm{PF}_{6}\right]$ (bottom).

this trend is not completely uniform across the table, the volume of hexafluorophosphates being better reproduced than that of the chlorides, especially by the $\kappa=1$ functional. This would suggest that the dispersion interaction is more important in the chlorides.

The difference between the two series is puzzling because one would expect a larger electrostatic contribution from the stronger $\mathrm{Cl}^{-}$anion, and thus a less relevant VDW contribution. A possible explanation can be found in Table IV, where we compare some selected intermolecular distances in $[\mathrm{mmim}][\mathrm{Cl}]$ and $[\mathrm{mmim}]\left[\mathrm{PF}_{6}\right]$.

Since the $\mathrm{Cl}^{-}$anion is smaller than $\mathrm{PF}_{6}^{-}$, the intermolecular distances are shorter in the chlorides, and thus the VDW energy contributions are larger. This is confirmed in Table VII, where we report the specific volumes (volume per neutral ion pair), which are clearly smaller in the chlorides for equivalent cations. For example, the $\mathrm{C}_{1^{\prime}}-\mathrm{C}_{1^{\prime}}$ distance between the $\mathrm{C}$-atoms in the methyl groups of the cation and the $\mathrm{C}_{4}-\mathrm{C}_{4}$ distance between the ring carbons are 0.6 and 1.15 Ashorter in $[\mathrm{mmim}][\mathrm{Cl}]$, which amounts to a substantial difference in VDW energy.

It is also interesting to compare the performance of the DRSLL functional with respect to GGA-PBE results in the $\mathrm{PF}_{6}$ series. For the first three systems, where the VDW interactions are less important because the alkyl side chain is shorter, the GGA-PBE error is around 12\%-13\%. However, for $[\mathrm{ddmim}]\left[\mathrm{PF}_{6}\right]$, which has a much longer alkyl group and thus more substantial VDW interactions, the error in volume increases up to a substantial $17 \%$, while the DRSLL and $\kappa$ $=1$ functionals do not degrade along the series, remaining around average values of $7.5 \%$ and $2.1 \%$, respectively. This is consistent with the observation that GGA functionals are grossly in error for long hydrocarbon chains. ${ }^{36}$ Therefore, we can conclude that the DRSLL functional constitutes a major improvement over both the LDA and GGA-PBE.

Even better $T=0 \mathrm{~K}$ volumes are obtained with the $\kappa=1$ functional, i.e., the DRSLL functional with a modified semilocal exchange. ${ }^{37}$ This suggests that the optimization of the $\kappa$ parameter in the PBE exchange could be a desirable feature, which is compatible with theoretical bounds and improves the description of non-bonded interactions, not only in the present systems but also in hydrogen-bonded systems such as water clusters. ${ }^{15}$ Further confirmation of the enhanced performance of the dispersion-corrected functionals is evident when comparing the equilibrium cell parameters, as shown in Table III. Reproduction of the lattice parameters is a more rigorous test of the performance of the functionals, and it is clear that the $\kappa=1$ functional is also superior in this regard-calculated RMSE over all cell lengths and angles is as small as $1.4 \%$.

This claim is also supported by a generally superior agreement with experiment in terms of internal geometries. Intra-molecular distances and angles are reproduced equally well by all four functionals, thus confirming that the VDW functionals do not introduce any unwanted features in the description of covalent bonds. For inter-molecular distances, the improvement is substantial, as can be judged from Tables V and VI. The last column reports the average relative error of these distances. The overall conclusion is that the agreement in the internal geometry is intimately connected to the

TABLE II. Zero-pressure equilibrium volume $\left(\AA^{3}\right)$ of the imidazolium-based crystals. The second column indicates the number of formula units in the unit cell $(Z)$. Columns three to six present the volumes obtained using the LDA, PBE, DRSLL, and $\kappa=1$ functionals, respectively. The last two columns report the extrapolated and measured experimental volumes. ${ }^{9}$ The average relative error of each approximation with respect to the extrapolated experimental volume is reported the last line.

\begin{tabular}{|c|c|c|c|c|c|c|c|}
\hline System & $Z$ & LDA & GGA-PBE & DRSLL & $\kappa=1$ & Exp. $(0 \mathrm{~K})$ & Exp. \\
\hline$[\mathrm{mmim}]\left[\mathrm{PF}_{6}\right]$ & 8 & 1638.4 & 2065.2 & 1983.9 & 1886.0 & 1830.3 & 1893.9 \\
\hline$[\mathrm{emim}]\left[\mathrm{PF}_{6}\right]$ & 4 & 895.1 & 1136.3 & 1081.1 & 1028.9 & 1014.1 & 1023.9 \\
\hline$[\mathrm{bmim}]\left[\mathrm{PF}_{6}\right]$ & 2 & 523.5 & 663.8 & 634.5 & 603.1 & 590.4 & 605.0 \\
\hline$[\mathrm{ddmim}]\left[\mathrm{PF}_{6}\right]$ & 8 & 1761.6 & 2294.1 & 2113.1 & 1998.9 & 1962.5 & 2000.6 \\
\hline Error $(\%)$ & & -10.9 & +13.5 & +7.5 & +2.1 & - & - \\
\hline$\overline{[\mathrm{mmim}][\mathrm{Cl}]}$ & 4 & 598.8 & 735.5 & 730.6 & 700.8 & 674.5 & 687.6 \\
\hline$[\mathrm{bmim}][\mathrm{Cl}]-\mathrm{o}$ & 4 & 848.7 & 1034.8 & 1023.8 & 985.2 & 947.3 & 961.1 \\
\hline$[\mathrm{bmim}][\mathrm{Cl}]-\mathrm{m}$ & 4 & 848.0 & 1047.8 & 1026.6 & 985.0 & 948.1 & 966.7 \\
\hline Error $(\%)$ & & -10.7 & +9.6 & +8.2 & +3.9 & - & - \\
\hline
\end{tabular}


TABLE III. Zero-pressure equilibrium cell lengths ( $)$ and angles (deg) of the imidazolium-based crystals.

\begin{tabular}{|c|c|c|c|c|c|}
\hline System & Method & $a$ & $b$ & $c$ & $\beta$ or $\alpha, \beta, \gamma$ \\
\hline \multirow{5}{*}[\mathrm{mmim}]{$\left[\mathrm{PF}_{6}\right]$} & LDA & 10.660 & 8.791 & 17.483 & 90.00 \\
\hline & PBE & 11.600 & 9.707 & 18.341 & 90.00 \\
\hline & DRSLL & 11.276 & 9.567 & 18.391 & 90.00 \\
\hline & $\kappa=1$ & 11.055 & 9.381 & 18.186 & 90.00 \\
\hline & Expt. $^{40}$ & 11.302 & 9.337 & 17.947 & 90.00 \\
\hline \multirow{5}{*}[\mathrm{emim}]{$\left[\mathrm{PF}_{6}\right]$} & LDA & 8.273 & 8.412 & 13.069 & 100.21 \\
\hline & PBE & 8.803 & 9.522 & 13.826 & 101.37 \\
\hline & DRSLL & 8.854 & 9.086 & 13.690 & 101.02 \\
\hline & $\kappa=1$ & 8.728 & 8.945 & 13.438 & 101.27 \\
\hline & Expt. ${ }^{41}$ & 8.617 & 9.016 & 13.458 & 101.68 \\
\hline \multirow{5}{*}[\mathrm{bmim}]{$\left[\mathrm{PF}_{6}\right]$} & LDA & 8.185 & 8.435 & 8.816 & $96.14,114.46,103.73$ \\
\hline & PBE & 8.945 & 9.227 & 9.177 & $94.43,114.03,102.47$ \\
\hline & DRSLL & 8.764 & 9.052 & 9.074 & $93.16,113.11,103.66$ \\
\hline & $\kappa=1$ & 8.568 & 8.877 & 8.996 & $92.51,113.44,103.54$ \\
\hline & Expt. $^{42}$ & 8.774 & 8.944 & 9.032 & $95.95,114.93,103.01$ \\
\hline \multirow{5}{*}[\mathrm{ddmim}]{$\left[\mathrm{PF}_{6}\right]$} & LDA & 8.910 & 9.355 & 21.156 & 92.62 \\
\hline & PBE & 9.744 & 10.324 & 22.994 & 97.36 \\
\hline & DRSLL & 9.297 & 10.139 & 22.463 & 93.65 \\
\hline & $\kappa=1$ & 9.134 & 9.908 & 22.118 & 93.07 \\
\hline & Expt. $^{43}$ & 9.1750 & 9.849 & 22.197 & 94.13 \\
\hline \multirow{5}{*}[\mathrm{mmim}]{$[\mathrm{Cl}]$} & LDA & 8.454 & 7.344 & 10.086 & 107.03 \\
\hline & PBE & 8.694 & 8.184 & 10.829 & 107.31 \\
\hline & DRSLL & 8.870 & 7.902 & 10.867 & 106.40 \\
\hline & $\kappa=1$ & 8.827 & 7.719 & 10.730 & 106.54 \\
\hline & Expt. $^{38}$ & 8.652 & 7.858 & 10.539 & 106.34 \\
\hline \multirow{5}{*}[\mathrm{bmim}]{$[\mathrm{Cl}-\mathrm{m}]$} & LDA & 9.479 & 11.196 & 8.952 & 116.81 \\
\hline & PBE & 10.184 & 11.840 & 9.913 & 118.76 \\
\hline & DRSLL & 10.046 & 11.942 & 9.690 & 117.98 \\
\hline & $\kappa=1$ & 9.877 & 11.786 & 9.538 & 117.49 \\
\hline & Expt. $^{39}$ & 9.943 & 11.481 & 9.658 & 118.74 \\
\hline \multirow{5}{*}[\mathrm{bmim}]{$[\mathrm{Cl}]-\mathrm{o}$} & LDA & 9.725 & 10.909 & 8.000 & 90.00 \\
\hline & PBE & 10.363 & 11.648 & 8.573 & 90.00 \\
\hline & DRSLL & 10.243 & 11.706 & 8.538 & 90.00 \\
\hline & $\kappa=1$ & 10.085 & 11.578 & 8.438 & 90.00 \\
\hline & Expt. $^{39}$ & 10.113 & 11.411 & 8.329 & 90.00 \\
\hline
\end{tabular}

agreement in volume and lattice parameters, as already suggested in Ref. 9. Unlike volumes, the extrapolation of experimental internal geometries to $T=0 \mathrm{~K}$ is ambiguous. Therefore, we compare to geometries directly measured at the experimental temperature, which correspond to the volume reported in the last column of Table II.

In the case of $[\mathrm{bmim}]\left[\mathrm{PF}_{6}\right]$ the best agreement is obtained with the original DRSLL functional, while PBE and $\kappa=1$

TABLE IV. Selected intermolecular bond lengths $(\AA)$ in $[\mathrm{mmim}][\mathrm{Cl}]$ and $[\mathrm{mmim}]\left[\mathrm{PF}_{6}\right]$.

\begin{tabular}{lcccccccc}
\hline \hline \multirow{2}{*}{ Bond } & \multicolumn{2}{c}{$\mathrm{C}_{4}-\mathrm{C}_{4}$} & & \multicolumn{2}{c}{$\mathrm{C}_{1^{\prime}}-\mathrm{C}_{1^{\prime}}$} & & \multicolumn{2}{c}{$\mathrm{C}_{2}-\mathrm{C}_{1^{\prime}}$} \\
\cline { 2 - 3 } & {$[\mathrm{Cl}]$} & {$\left[\mathrm{PF}_{6}\right]$} & & {$[\mathrm{Cl}]$} & {$\left[\mathrm{PF}_{6}\right]$} & & {$[\mathrm{Cl}]$} & {$\left[\mathrm{PF}_{6}\right]$} \\
\hline LDA & 3.05 & 4.38 & & 3.43 & 4.15 & & 3.32 & 3.51 \\
PBE & 3.34 & 4.88 & & 3.89 & 4.54 & & 3.57 & 4.04 \\
DRSLL & 3.35 & 4.79 & & 3.83 & 4.46 & & 3.55 & 3.89 \\
$\kappa=1$ & 3.30 & 4.70 & & 3.74 & 4.36 & & 3.50 & 3.78 \\
Exp. & 2.90 & 4.06 & & 3.60 & 4.20 & & 3.87 & 3.83 \\
\hline \hline
\end{tabular}

are of similar quality, which is somewhat puzzling. We would expect that the functional which reproduces the volume more closely $(\kappa=1)$ would also produce the best internal geometry. The trend is, however, clear; as volume decreases from PBE to DRSLL and $\kappa=1$, distances also decrease, going through a particularly good agreement at the DRSLL volume. A closer inspection shows that distances involving the $\mathrm{P}$ atoms are the ones in larger disagreement for $\kappa=1$, and this is also the case of $[\mathrm{ddmim}]\left[\mathrm{PF}_{6}\right]$ (see Table VI). The distances between $\mathrm{C}$ atoms in neighbouring cations are reproduced to similar extents by the three functionals, while the LDA is clearly in error. Empirical VDW corrections of the form Eq. (1) as proposed in Ref. 9, which are constructed in such a way as to reproduce the experimental, finite-temperature volume, produce intra-molecular distances of quality inferior to the nonempirical ones studied here.

By increasing the length of the alkyl group as in [ddmim] $\left[\mathrm{PF}_{6}\right]$, the quality of the PBE functional degrades to levels comparable to those of the LDA. The DRSLL and $\kappa$ $=1$ functionals, however, retain a similar level of 
TABLE V. Selected intermolecular bond lengths $(\AA)$ in $[\mathrm{bmim}]\left[\mathrm{PF}_{6}\right]$. Experimental data from Ref. 42. Numbers in parentheses indicate the percentage difference between calculated and experimental distances. The last column lists the average error.

\begin{tabular}{|c|c|c|c|c|c|c|c|c|c|c|}
\hline Bond & $\mathrm{C}_{5}-\mathrm{C}_{5}$ & $\mathrm{C}_{1^{\prime \prime}}-\mathrm{C}_{1^{\prime \prime}}$ & $\mathrm{C}_{4^{\prime}}-\mathrm{C}_{4^{\prime}}$ & $\mathrm{C}_{2}-\mathrm{C}_{4^{\prime}}$ & $\mathrm{C}_{4^{\prime}}-\mathrm{C}_{1^{\prime \prime}}$ & P-P & $\mathrm{C}_{2}-\mathrm{P}$ & $\mathrm{C}_{1^{\prime \prime}}-\mathrm{P}$ & $\mathrm{C}_{4^{\prime}}-\mathrm{P}$ & Error $(\%)$ \\
\hline LDA & $\begin{array}{c}4.73 \\
(-7.2)\end{array}$ & $\begin{array}{c}4.22 \\
(-0.7)\end{array}$ & $\begin{array}{c}3.78 \\
(-6.6)\end{array}$ & $\begin{array}{c}4.66 \\
(-5.3)\end{array}$ & $\begin{array}{c}3.68 \\
(-4.7)\end{array}$ & $\begin{array}{c}5.12 \\
(-9.5)\end{array}$ & $\begin{array}{c}3.75 \\
(-5.0)\end{array}$ & $\begin{array}{c}3.90 \\
(-9.0)\end{array}$ & $\begin{array}{c}4.82 \\
(-2.4)\end{array}$ & (5.6) \\
\hline PBE & $\begin{array}{c}5.27 \\
(+3.3)\end{array}$ & $\begin{array}{c}4.88 \\
(+8.0)\end{array}$ & $\begin{array}{c}4.02 \\
(-0.7)\end{array}$ & $\begin{array}{c}4.98 \\
(+1.2)\end{array}$ & $\begin{array}{c}4.06 \\
(+5.2)\end{array}$ & $\begin{array}{c}5.90 \\
(+4.2)\end{array}$ & $\begin{array}{c}4.00 \\
(+1.2)\end{array}$ & $\begin{array}{c}4.30 \\
(+0.2)\end{array}$ & $\begin{array}{c}4.98 \\
(+0.8)\end{array}$ & (2.8) \\
\hline DRSLL & $\begin{array}{c}5.13 \\
(+0.6)\end{array}$ & $\begin{array}{c}4.58 \\
(+1.3)\end{array}$ & $\begin{array}{c}4.06 \\
(+0.2)\end{array}$ & $\begin{array}{c}4.84 \\
(-1.6)\end{array}$ & $\begin{array}{c}3.97 \\
(+2.8)\end{array}$ & $\begin{array}{c}5.64 \\
(-0.4)\end{array}$ & $\begin{array}{c}3.93 \\
(-0.5)\end{array}$ & $\begin{array}{c}4.16 \\
(-3.0)\end{array}$ & $\begin{array}{c}4.95 \\
(+0.2)\end{array}$ & $(1.2)$ \\
\hline$\kappa=1$ & $\begin{array}{c}4.98 \\
(-2.4)\end{array}$ & $\begin{array}{c}4.43 \\
(-1.9)\end{array}$ & $\begin{array}{c}4.02 \\
(-0.7)\end{array}$ & $\begin{array}{c}4.74 \\
(-3.7)\end{array}$ & $\begin{array}{l}3.86 \\
(0.0)\end{array}$ & $\begin{array}{c}5.45 \\
(-3.7)\end{array}$ & $\begin{array}{c}3.82 \\
(-3.3)\end{array}$ & $\begin{array}{c}4.07 \\
(-5.1)\end{array}$ & $\begin{array}{c}4.89 \\
(-1.0)\end{array}$ & (2.4) \\
\hline Exp. & 5.10 & 4.52 & 4.05 & 4.92 & 3.86 & 5.66 & 3.95 & 4.29 & 4.94 & \\
\hline
\end{tabular}

performance with an average error in inter-molecular distances of $\approx 2 \%$. This is again consistent with the behaviour of the volumes, the error in the distances generally changing sign as volume decreases in going from DRSLL to $\kappa=1$.

Therefore, it appears that the concerted improvement of both non-local correlation and semi-local exchange are necessary ingredients to reach a desirable $1 \%-2 \%$ level of accuracy in lattice parameters and internal geometries. While lattice parameters require both ingredients, inter-molecular distances are dramatically improved by the VDW non-local correlation alone, irrespective of the flavor of the GGA exchange utilized.

The other major feature addressed by the DRSLL functional is of energetic character. It is well-known that, due to the lack of dispersion interactions, the GGA severely underbinds systems such as those in the present case. The LDA also lacks dispersion interactions, but this is often fortuitously compensated by its tendency to produce more uniform charge distributions leading to overbinding. We computed the cohesive energies for the two series of crystals and compared the results of the various functionals and of the empirical force field of Ref. 3. Results are presented in Table VII. A noticeable feature (last column) is the decrease of the energy density $\epsilon$ with the length of the side chain. While the cohesive energy per ionic pair tends to increase along the series, the specific volume also increases together with the size of the cation. The cohesive energies include an electrostatic contribution that decreases with increasing specific volume, while dispersion interactions are enhanced. In balance, this competition causes the cohesive energy to grow more slowly than the volume, thus producing the observed trend.
According to the findings for rare gas dimers and related systems, the original DRSLL functional is expected to overbind. In the present systems, due to the large unit cells, it has not been possible to compute binding energies at a higher level such as Møller-Plesset perturbation theory. In addition, experimental data on cohesive energies is not available. We therefore took the $\kappa=1$ values as reference and compared the other functionals and the empirical force field of Ref. 3 with it. First of all, the PBE functional produces cohesive energies that are less than half the VDW ones, thus confirming its tendency to severely underbind. The LDA compensates due to the smaller volume, although in the $\mathrm{PF}_{6}$ series it still underbinds significantly. In the $\mathrm{Cl}$ series, the LDA reproduces quite closely the DRSLL energies, but still underbinds relative to $\kappa=1$. In effect, increasing the value of the parameter $\kappa$ in the VDW functional has the effect of enhancing the exchange energy, thus increasing the cohesive energy in $\approx 10 \%\left(15 \%\right.$ in $\left.[\mathrm{ddmim}]\left[\mathrm{PF}_{6}\right]\right)$. The cohesive energies produced by the force field (FF) are close to those obtained with the $\kappa=1$ functional, with the exception of [ddmim $]\left[\mathrm{PF}_{6}\right]$ and $[\mathrm{mmim}][\mathrm{Cl}]$, whose cohesive energy the FF underestimates (overestimates) by $14 \%$ (20\%). In any case, these energies are within the expected accuracy of the VDW functionals, thus justifying the previous and future use of carefully constructed force fields such as those of Ref. 3 .

\section{B. Polymorphism of $[\mathrm{bmim}][\mathrm{Cl}]$}

Two different crystalline forms of [bmim][Cl] have been identified in experiments, of monoclinic and orthorhombic symmetries. In one of the experiments, carried out at

TABLE VI. Selected intermolecular bond lengths $(\AA)$ in $[\mathrm{ddmim}]\left[\mathrm{PF}_{6}\right]$. Experimental data from Ref. 43. Numbers in parentheses indicate the percentage difference between calculated and experimental distances. Last column has the average error.

\begin{tabular}{|c|c|c|c|c|c|c|c|c|c|c|c|c|}
\hline Bond & $\mathrm{C}_{4}-\mathrm{C}_{4}$ & $\mathrm{C}_{1^{\prime \prime}}-\mathrm{C}_{1^{\prime \prime}}$ & $\mathrm{C}_{12^{\prime}}-\mathrm{C}_{12^{\prime}}$ & $\mathrm{C}_{4^{\prime}}-\mathrm{C}_{4^{\prime}}$ & $\mathrm{C}_{2}-\mathrm{C}_{4^{\prime}}$ & $\mathrm{C}_{4^{\prime}}-\mathrm{C}_{1^{\prime \prime}}$ & P-P & $\mathrm{C}_{2}-\mathrm{P}$ & $\mathrm{C}_{1^{\prime \prime}}-\mathrm{P}$ & $\mathrm{C}_{4^{\prime}}-\mathrm{P}$ & $\mathrm{C}_{12^{\prime}}-\mathrm{P}$ & Error (\%) \\
\hline LDA & $\begin{array}{c}4.58 \\
(-2.3)\end{array}$ & $\begin{array}{c}4.04 \\
(-7.3)\end{array}$ & $\begin{array}{c}4.95 \\
(-2.8)\end{array}$ & $\begin{array}{c}4.67 \\
(-3.7)\end{array}$ & $\begin{array}{c}4.31 \\
(-6.5)\end{array}$ & $\begin{array}{c}6.01 \\
(-3.1)\end{array}$ & $\begin{array}{c}6.25 \\
(+4.0)\end{array}$ & $\begin{array}{c}3.59 \\
(-5.0)\end{array}$ & $\begin{array}{c}3.96 \\
(-1.2)\end{array}$ & $\begin{array}{c}4.27 \\
(-7.6)\end{array}$ & $\begin{array}{c}4.41 \\
(-4.8)\end{array}$ & (5.1) \\
\hline PBE & $\begin{array}{c}5.15 \\
(+9.8)\end{array}$ & $\begin{array}{c}4.26 \\
(-2.3)\end{array}$ & $\begin{array}{c}5.44 \\
(+6.9)\end{array}$ & $\begin{array}{c}5.11 \\
(+5.4)\end{array}$ & $\begin{array}{c}5.13 \\
(+11.3)\end{array}$ & $\begin{array}{c}6.64 \\
(+2.9)\end{array}$ & $\begin{array}{c}6.80 \\
(+5.0)\end{array}$ & $\begin{array}{c}3.92 \\
(+3.7)\end{array}$ & $\begin{array}{c}4.31 \\
(+7.5)\end{array}$ & $\begin{array}{c}5.08 \\
(+9.9)\end{array}$ & $\begin{array}{c}4.80 \\
(+3.0)\end{array}$ & (6.1) \\
\hline DRSLL & $\begin{array}{c}5.06 \\
(+7.9)\end{array}$ & $\begin{array}{c}4.45 \\
(+2.0)\end{array}$ & $\begin{array}{c}5.12 \\
(+0.6)\end{array}$ & $\begin{array}{c}4.92 \\
(+1.4)\end{array}$ & $\begin{array}{c}4.70 \\
(+1.9)\end{array}$ & $\begin{array}{c}6.42 \\
(-0.5)\end{array}$ & $\begin{array}{c}6.58 \\
(+1.2)\end{array}$ & $\begin{array}{c}3.85 \\
(+1.9)\end{array}$ & $\begin{array}{c}4.23 \\
(+5.5)\end{array}$ & $\begin{array}{c}4.63 \\
(+0.2)\end{array}$ & $\begin{array}{c}4.67 \\
(+0.9)\end{array}$ & (2.2) \\
\hline$\kappa=1$ & $\begin{array}{c}4.86 \\
(+3.6)\end{array}$ & $\begin{array}{c}4.36 \\
(+0.0)\end{array}$ & $\begin{array}{c}5.03 \\
(-1.2)\end{array}$ & $\begin{array}{c}4.84 \\
(-0.2)\end{array}$ & $\begin{array}{c}4.55 \\
(-1.3)\end{array}$ & $\begin{array}{c}6.30 \\
(-2.3)\end{array}$ & $\begin{array}{c}6.43 \\
(-1.4)\end{array}$ & $\begin{array}{l}3.78 \\
(0.0)\end{array}$ & $\begin{array}{c}4.20 \\
(+4.7)\end{array}$ & $\begin{array}{c}4.44 \\
(-3.9)\end{array}$ & $\begin{array}{c}4.58 \\
(-1.1)\end{array}$ & (1.8) \\
\hline Exp. & 4.69 & 4.36 & 5.09 & 4.85 & 4.61 & 6.25 & 6.45 & 3.78 & 4.01 & 4.62 & 4.63 & $\ldots$ \\
\hline
\end{tabular}


TABLE VII. Cohesive energies (eV/pair) of the imidazolium-based crystals. Columns two to six present DFT results using the LDA, PBE, DRSLL, and $\kappa=1$ functionals, and the force field (FF) of Ref. 3, respectively. The last two columns report the specific volume (volume per neutral ion pair) $\left(\AA^{3} /\right.$ pair $)$ and energy density $\epsilon\left(\mathrm{eV} / \AA^{3}\right)$ obtained with the $\kappa=1$ functional.

\begin{tabular}{lccccccc}
\hline \hline System & LDA & PBE & DRSLL & $\kappa=1$ & FF & Vol & $\epsilon \times 10^{-3}$ \\
\hline$\left[\mathrm{mmim}_{[}\right]\left[\mathrm{PF}_{6}\right]$ & 1.79 & 1.16 & 1.94 & $\mathbf{2 . 2 0}$ & 2.26 & 248.0 & 8.9 \\
{$[\mathrm{emim}]\left[\mathrm{PF}_{6}\right]$} & 1.78 & 1.12 & 2.06 & $\mathbf{2 . 3 0}$ & 2.30 & 270.3 & 8.5 \\
[bmim $]\left[\mathrm{PF}_{6}\right]$ & 1.89 & 1.00 & 2.04 & $\mathbf{2 . 3 5}$ & 2.24 & 317.2 & 7.4 \\
{$[$ ddmim $]\left[\mathrm{PF}_{6}\right]$} & 2.41 & 1.17 & 2.76 & $\mathbf{3 . 2 5}$ & 2.80 & 528.3 & 6.2 \\
{$[\mathrm{mmim}][\mathrm{Cl}]$} & 1.93 & 1.18 & 1.77 & $\mathbf{1 . 9 9}$ & 2.39 & 182.7 & 10.9 \\
{$[$ bmim $][\mathrm{Cl}]-\mathrm{m}$} & 1.98 & 1.10 & 2.00 & $\mathbf{2 . 2 7}$ & 2.40 & 256.7 & 8.8 \\
{$[$ bmim $][\mathrm{Cl}]-\mathrm{o}$} & 1.94 & 1.04 & 2.00 & $\mathbf{2 . 2 7}$ & 2.48 & 256.0 & 8.9 \\
\hline \hline
\end{tabular}

$T=173 \mathrm{~K}$, it was claimed that the orthorhombic structure was the stable one, while the monoclinic one appeared as a metastable state under certain external conditions ([bmim $][\mathrm{Cl}]$ in Ref. 35). The opposite picture was proposed in Ref. 44, namely that the monoclinic structure was stable and the orthorhombic one metastable. These contrasting interpretations led to a controversy that is still open, and also to the notion that this system may actually offer different polymorphic alternatives for the crystalline phase. Previous computational work was inconclusive in this respect. While both structures were found to be locally stable and conforming to the experimental crystallographic information, the cohesive energy difference between them was found to favor the monoclinic phase by 0.12 and $0.16 \mathrm{eV} /$ pair in the LDA and GGA-PBE, respectively. ${ }^{9}, 45$ On the contrary, the force field of Canongia Lopes $\mathrm{et} \mathrm{al.}{ }^{3}$ produced the opposite result, namely that the orthorhombic phase was more stable by $0.08 \mathrm{eV} /$ pair, and computations of the Gibbs free energy for increasing temperature did not show any indication of a possible phase transition towards the monoclinic structure. ${ }^{9}$ A contrasting classical MD study using a different force field produced the intriguing result that the free energy difference between the two polymorphs was vary small, and thus it was not possible to establish which of the two structures was more stable. ${ }^{46}$

In order to shed more light on this controversy, we computed the energy difference between the two structures using the DRSLL functional. In addition, we have re-computed the energy difference for LDA and PBE using the improved basis set. The previous results for LDA and PBE hold, but the energy differences are 0.04 and $0.06 \mathrm{eV}$, respectively. For both VDW functionals, we observe a surprisingly small energy difference, smaller than $0.01 \mathrm{eV} /$ pair, favoring the monoclinic structure in agreement with the other two functionals. This energy difference, however, is sufficiently small as to be affected by neglected contributions such as zero-point energy or volume effects (recalling the fact that DRSLL and $\kappa=1$ still overestimate volumes by $8 \%$ and $4 \%$, respectively). In any event, the small energy difference suggests a possible polymorphism. As they stand, these results support the claims of Ref. 44 but, due to the small energy difference, the possibility of a phase transition between the two structures upon increasing temperature is re-instated, in agreement with the classical force field results of Jayaraman and Maginn. ${ }^{46}$
Electronic energy gaps at the $\Gamma$-point of the Brillouin zone are similar for the two structures. LDA, GGA-PBE, and VDW energy gaps are larger in the monoclinic structure, consistently with a higher stability, but the difference is only about $0.2 \mathrm{eV}$. DRSLL gaps are slightly smaller than GGA ones but still in excess of $3 \mathrm{eV}$, so that $[\mathrm{bmim}][\mathrm{Cl}]$ remains a wide gap insulator. Band gaps are considerably understimated with respect to experiment by all the functionals considered here. The above concerns only the comparison between structures.

In Sec. III A, we have established that the DRSLL functional tends to describe intermolecular geometries better than local and semi-local functionals. In Table VIII, we compare intermolecular distances in the two structures for all four functionals, and we confirm that the VDW functionals perform significantly better. An improvement in intermolecular distances is observed with DRSLL, and especially the $\kappa$ $=1$ functional, in comparison with experiment. The most significant improvement is in the distances between $\mathrm{C}$-atoms in the alkyl chains. On average, $\kappa=1$ distances are within $\approx 1$ $\%$ of the experimental values. This general improvement of the VDW geometry could explain the change in the energy difference between the two structures relative to LDA and GGA-PBE, thus supporting the previous interpretation that was based on energetic considerations.

\section{Structure and energetics of imidazolium-based clusters}

It has been argued in Ref. 9 that departures in intermolecular distances and angles observed for the LDA and GGA$\mathrm{PBE}$ are mostly due to their poor performance in reproducing experimental volumes. If the internal geometry is optimized at fixed lattice parameters taken from experiment, the agreement with experimental values is much better. This is certainly useful if experimental crystallographic information is available, but otherwise represents a problem. This acquires particular relevance when studying the properties of aggregates, as was done in Ref. 14 for [bmim][Tf] (Tf is shorthand for the triflate anion, $\mathrm{SO}_{3} \mathrm{CF}_{3}^{-}$) and in Ref. 47 for [mmim] [Cl], because there is no experimental information about their structure.

\section{1. [bmim $][T f]$}

In Ref. 14, the main goal was to study the composition of the vapour phase of [bmim][Tf]. The motivation for this work was that, despite the widespread statement that ionic liquids do not evaporate, and thus do not contribute to air pollution, under certain high-temperature low-pressure conditions they were successfully distilled by evaporation. ${ }^{48}$ Computational study required the determination of the structure of clusters of increasing size as well as the corresponding positively and negatively charged ions. Given the known limitations of both the LDA and the GGA-PBE in describing dispersion interactions adequately, it was considered that the safest approach was to use a carefully parameterized classical force field, rather than first-principles simulations. ${ }^{3}$ This strategy was also adopted because, for larger clusters, the exploration of the energy landscape from the structural point of 
TABLE VIII. Selected intermolecular distances $(\AA)$ in $[\mathrm{bmim}][\mathrm{Cl}]$ in its monoclinic and orthorhombic forms. For each pair of atoms we report the separation between atoms whose distance is the shortest in the experimental structure. Numbers in parentheses indicate the percentage difference between calculated and experimental distances.

\begin{tabular}{|c|c|c|c|c|c|c|c|c|c|}
\hline \multirow[b]{2}{*}{ Bond } & \multirow[b]{2}{*}{$\mathrm{C}_{5}-\mathrm{C}_{5}$} & \multirow[b]{2}{*}{$\mathrm{C}_{1^{\prime \prime}}-\mathrm{C}_{1^{\prime \prime}}$} & \multicolumn{3}{|c|}{$[\mathrm{bmim}][\mathrm{Cl}]-$ monoclinic } & \multirow[b]{2}{*}{$\mathrm{C}_{2}-\mathrm{Cl}$} & \multirow[b]{2}{*}{$\mathrm{C}_{1^{\prime \prime}}-\mathrm{Cl}$} & \multirow[b]{2}{*}{$\mathrm{C}_{4^{\prime}}-\mathrm{Cl}$} & \multirow[b]{2}{*}{ Error $(\%)$} \\
\hline & & & $\mathrm{C}_{4^{\prime}}-\mathrm{C}_{4^{\prime}}$ & $\mathrm{C}_{2}-\mathrm{C}_{4^{\prime}}$ & $\mathrm{C}_{4^{\prime}}-\mathrm{C}_{1^{\prime \prime}}$ & & & & \\
\hline LDA & $\begin{array}{c}4.94 \\
(-4.8)\end{array}$ & $\begin{array}{c}4.60 \\
(-3.8)\end{array}$ & $\begin{array}{c}5.73 \\
(-5.8)\end{array}$ & $\begin{array}{c}3.27 \\
(-6.0)\end{array}$ & $\begin{array}{c}3.79 \\
(+4.7)\end{array}$ & $\begin{array}{c}3.28 \\
(-3.2)\end{array}$ & $\begin{array}{c}3.51 \\
(-8.1)\end{array}$ & $\begin{array}{c}3.69 \\
(-2.9)\end{array}$ & $(4.9)$ \\
\hline PBE & $\begin{array}{c}5.25 \\
(+1.2)\end{array}$ & $\begin{array}{c}4.70 \\
(-1.7)\end{array}$ & $\begin{array}{c}5.89 \\
(-3.1)\end{array}$ & $\begin{array}{c}3.62 \\
(+4.0)\end{array}$ & $\begin{array}{c}3.78 \\
(+4.4)\end{array}$ & $\begin{array}{c}3.40 \\
(+0.3)\end{array}$ & $\begin{array}{c}3.84 \\
(+0.5)\end{array}$ & $\begin{array}{c}3.89 \\
(+2.4)\end{array}$ & (2.2) \\
\hline DRSLL & $\begin{array}{c}5.22 \\
(+0.6)\end{array}$ & $\begin{array}{c}4.97 \\
(+4.0)\end{array}$ & $\begin{array}{c}6.17 \\
(+1.5)\end{array}$ & $\begin{array}{c}3.52 \\
(+1.1)\end{array}$ & $\begin{array}{c}3.74 \\
(+3.3)\end{array}$ & $\begin{array}{c}3.47 \\
(+2.4)\end{array}$ & $\begin{array}{c}3.85 \\
(+0.8)\end{array}$ & $\begin{array}{c}3.96 \\
(+4.2)\end{array}$ & $(2.2)$ \\
\hline$\kappa=1$ & $\begin{array}{c}5.16 \\
(-0.6)\end{array}$ & $\begin{array}{c}4.89 \\
(-2.3)\end{array}$ & $\begin{array}{c}6.11 \\
(+0.5)\end{array}$ & $\begin{array}{c}3.45 \\
(-0.9)\end{array}$ & $\begin{array}{c}3.68 \\
(+1.7)\end{array}$ & $\begin{array}{c}3.44 \\
(+1.5)\end{array}$ & $\begin{array}{c}3.78 \\
(-1.0)\end{array}$ & $\begin{array}{c}3.89 \\
(+2.4)\end{array}$ & (1.4) \\
\hline \multicolumn{10}{|c|}{$[\mathrm{bmim}][\mathrm{Cl}]$ —orthorhombic } \\
\hline Bond & $\mathrm{C}_{5}-\mathrm{C}_{5}$ & $\mathrm{C}_{1^{\prime \prime}}-\mathrm{C}_{1^{\prime \prime}}$ & $\mathrm{C}_{4^{\prime}}-\mathrm{C}_{4^{\prime}}$ & $\mathrm{C}_{2}-\mathrm{C}_{4^{\prime}}$ & $\mathrm{C}_{4^{\prime}}-\mathrm{C}_{1^{\prime \prime}}$ & $\mathrm{C}_{2}-\mathrm{Cl}$ & $\mathrm{C}_{1^{\prime \prime}}-\mathrm{Cl}$ & $\mathrm{C}_{4^{\prime}}-\mathrm{Cl}$ & Error $(\%)$ \\
\hline LDA & $\begin{array}{c}5.20 \\
(-2.8)\end{array}$ & $\begin{array}{c}4.86 \\
(-4.1)\end{array}$ & $\begin{array}{c}4.44 \\
(+0.4)\end{array}$ & $\begin{array}{c}3.14 \\
(-7.6)\end{array}$ & $\begin{array}{c}3.54 \\
(-3.0)\end{array}$ & $\begin{array}{c}3.29 \\
(-2.9)\end{array}$ & $\begin{array}{c}3.40 \\
(-8.1)\end{array}$ & $\begin{array}{c}3.72 \\
(-8.4)\end{array}$ & (4.7) \\
\hline PBE & $\begin{array}{c}5.47 \\
(+2.2)\end{array}$ & $\begin{array}{c}5.19 \\
(+2.4)\end{array}$ & $\begin{array}{c}4.52 \\
(+2.3)\end{array}$ & $\begin{array}{c}3.48 \\
(+2.4)\end{array}$ & $\begin{array}{c}3.76 \\
(+3.0)\end{array}$ & $\begin{array}{c}3.42 \\
(+0.9)\end{array}$ & $\begin{array}{c}3.76 \\
(+1.6)\end{array}$ & $\begin{array}{c}4.08 \\
(-0.7)\end{array}$ & (1.9) \\
\hline DRSLL & $\begin{array}{c}5.42 \\
(+1.3)\end{array}$ & $\begin{array}{c}5.13 \\
(+1.2)\end{array}$ & $\begin{array}{c}4.56 \\
(+3.2)\end{array}$ & $\begin{array}{c}3.48 \\
(+2.4)\end{array}$ & $\begin{array}{c}3.68 \\
(+0.8)\end{array}$ & $\begin{array}{c}3.46 \\
(+2.1)\end{array}$ & $\begin{array}{c}3.68 \\
(+0.5)\end{array}$ & $\begin{array}{c}4.12 \\
(+1.7)\end{array}$ & (1.7) \\
\hline$\kappa=1$ & $\begin{array}{c}5.34 \\
(-0.2)\end{array}$ & $\begin{array}{c}5.05 \\
(-0.4)\end{array}$ & $\begin{array}{c}4.53 \\
(+2.5)\end{array}$ & $\begin{array}{c}3.41 \\
(+0.3)\end{array}$ & $\begin{array}{c}3.62 \\
(-0.8)\end{array}$ & $\begin{array}{c}3.43 \\
(+1.2)\end{array}$ & $\begin{array}{c}3.62 \\
(-2.2)\end{array}$ & $\begin{array}{l}4.06 \\
(0.2)\end{array}$ & (1.0) \\
\hline Exp. & 5.35 & 5.07 & 4.42 & 3.40 & 3.65 & 3.39 & 3.70 & 4.05 & \\
\hline
\end{tabular}

view is an extremely demanding task that requires clever algorithms and the evaluation of the energy for millions of configurations. In Fig. 3, we show the geometric arrangement in the ionic pair and dimer of pairs. Table IX summarizes some relevant inter-molecular distances in the ion pair. In this table, we include distances calculated at the second order MøllerPlesset perturbation level (MP2) and the $6-31 G^{*}$ basis set, obtained with the GAMESS-US code. ${ }^{49}$ In this section, we will consider MP2/6-31G* results as the target for DFT calculations.

While in Ref. 14, the relative energies and structures of a few small clusters were checked against LDA and GGA-PBE computations, this comparison was not particularly revealing, apart from confirming the accuracy of ionization energies obtained with the FF. This is not surprising, since most of that energy is of electrostatic origin, which is described equally accurately by DFT and FF approaches. Here we have com-
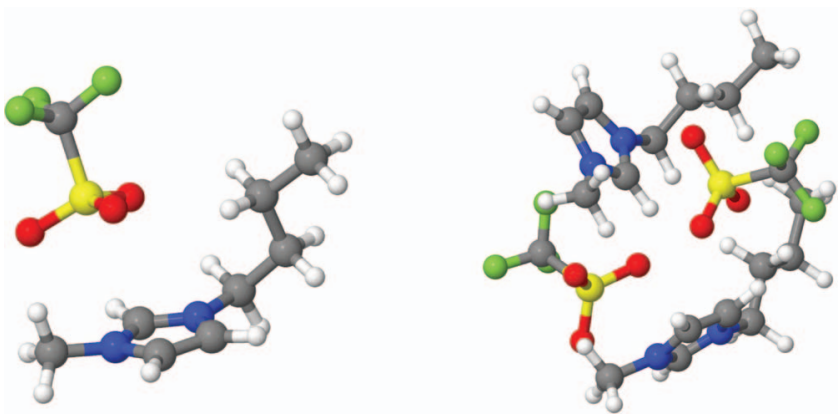

FIG. 3. Structures of the [bmim][Tf] ionic pair (left) and dimer of ionic pairs (right), optimized with the VDW functional. puted ionization energies, now using the DRSLL and $\kappa=1$ functionals, for which we obtained the values 4.0 and $4.2 \mathrm{eV}$, respectively. This is to be compared with $3.6 \mathrm{eV}$ obtained with the force field. ${ }^{14}$

A more stringent test is the comparison of pair binding energies and geometries; since the two fragments are neutral, electrostatics is less relevant and dispersion interactions play the dominant role. In Table $\mathrm{X}$, we report selected inter-molecular distances for the dimer, and the dimer binding energy for the LDA, GGA-PBE, DRSLL, and $\kappa$ $=1$ functionals as well as MP2 calculations and the FF results. While the two VDW functionals perform better for intramolecular distances, the results for the dimer do not improve over PBE, which is already quite close to MP2. This follows the same trend as for the solids. However, the PBE binding energy is quite small precisely due to the lack of dispersion interactions. The good agreement in geometries is probably due to the well known trend of GGA to underbind

TABLE IX. Selected intermolecular distances $(\AA)$ for the $[\mathrm{bmim}][\mathrm{Tf}]$ ion pair for the various density functionals and the force field of Ref. 3 . The quantum-chemical MP2/6-31G* results are taken as a higher level reference.

\begin{tabular}{lcccc}
\hline \hline & $\mathrm{S}-\mathrm{C}_{2}$ & $\mathrm{O} 1-\mathrm{C}_{3^{\prime}}$ & $\mathrm{O} 2-\mathrm{N}_{1}$ & $\mathrm{~S}-\mathrm{C}_{4^{\prime}}$ \\
\hline FF & 3.24 & 3.37 & 3.13 & 5.23 \\
LDA & 3.41 & 3.27 & 3.36 & 5.43 \\
PBE & 3.54 & 3.52 & 3.47 & 6.07 \\
DRSLL & 3.60 & 3.46 & 3.54 & 5.66 \\
$\kappa=1$ & 3.57 & 3.45 & 3.50 & 5.60 \\
MP2 & 3.56 & 3.51 & 3.54 & 5.59 \\
\hline \hline
\end{tabular}


TABLE X. Selected intermolecular distances $(\AA)$ and binding energy of the [bmim][Tf] dimer (in eV).

\begin{tabular}{lccccccc}
\hline \hline & $\mathrm{S}-\mathrm{S}$ & $\mathrm{C}_{2}-\mathrm{C}_{2}$ & $\mathrm{C}_{2}-\mathrm{S}$ & $\mathrm{S}-\mathrm{C}_{3^{\prime}}$ & $\mathrm{C}_{4^{\prime}}-\mathrm{C}_{4^{\prime}}$ & $\mathrm{C}_{2^{\prime}}-\mathrm{C}_{2^{\prime}}$ & $\Delta E$ \\
\hline FF & 5.93 & 4.92 & 3.45 & 4.48 & 5.11 & 4.82 & 1.41 \\
LDA & 5.34 & 4.80 & 3.32 & 5.09 & 3.97 & 4.64 & 1.36 \\
PBE & 6.02 & 5.11 & 3.47 & 4.90 & 6.28 & 5.26 & 0.86 \\
DRSLL & 6.06 & 5.09 & 3.52 & 4.65 & 5.91 & 5.09 & 1.39 \\
$\kappa=1$ & 6.03 & 4.98 & 3.49 & 4.61 & 5.73 & 5.01 & 1.53 \\
MP2 & 6.00 & 5.07 & 3.45 & 4.74 & 6.42 & 5.29 & 0.94 \\
\hline \hline
\end{tabular}

and overestimate distances. The FF seems to overbind with respect to MP2 as can be seen in distances and binding energy, but overall the FF is sufficiently accurate. We have employed this particular FF since it has been used in many previous studies. More accurate FFs including polarization are available in the literature, ${ }^{50}$ and study of their performance will be addressed in the future. The LDA, as usual, produces distances that are significantly shorter than all other approaches, including the FF. The $\mathrm{C}_{4^{\prime}}-\mathrm{C}_{4^{\prime}}$ distance appears to change considerably from one approach to another. In fact, small rotations of the alkyl chains produce amplified effects on the distances between the end of the chains.

\section{2. [mmim $][\mathrm{Cl}]$}

Previous calculations on $[\mathrm{mmim}][\mathrm{Cl}]$ (Ref. 5) have shown that the molecular ion pair has two isomers separated by an energy difference smaller than $0.14 \mathrm{eV}$. In the most stable configuration, the chloride ion is located above the plane of the imidazolium ring and placed on top of the $\mathrm{C}_{2}$ atom. Calculations performed using the united atom force field for $[\mathrm{mmim}][\mathrm{Cl}]$ developed by Price et al. ${ }^{51}$ agree with this. However, the isomer realized in the liquid phase is the other one, where the anion is hydrogen-bonded to the $\mathrm{C}_{2}$ carbon and located in the plane of the ring, where it can also interact with the $\mathrm{C}_{4}$ and $\mathrm{C}_{5}$ carbons of neighbouring cations. ${ }^{5}$ Force field calculations carried out using the set of parameters proposed by Youngs et al., ${ }^{6}$ where the hydrogens of the methyl groups are explicitly taken into account, reproduce this configuration and favor the formation of larger clusters characterized by low moments of inertia and small electric dipoles. ${ }^{47}$ This is in contrast with chain-like configurations obtained using the force field of Ref. 51, which were found to be energetically less stable at the first principles level. ${ }^{47}$ The structure of the dimer is shown in Fig. 4 for the DRSLL functional (left panel) and for the united atom force field (right panel).
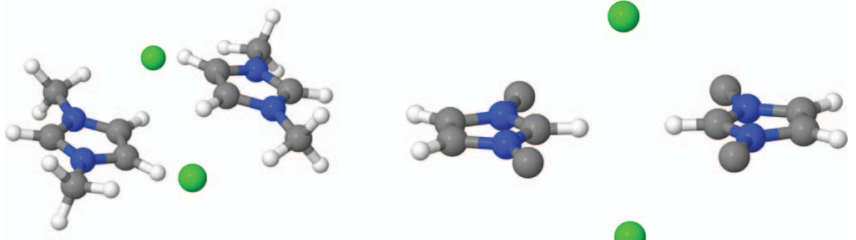

FIG. 4. Structures of the dimer of $[\mathrm{mmim}][\mathrm{Cl}]$ ionic pairs, optimized with the DRSLL functional (left panel), and with the force field of Ref. 51 that represents the methyl groups as united atoms (right panel). The force field of Ref. 6 produces clusters similar to those obtained with the VDW functionals.
TABLE XI. Selected bond lengths $(\AA)$ for the $[\mathrm{mmim}][\mathrm{Cl}]$ ion pair and dimer of pairs. $\Delta E$ is the dimer binding energy (in $\mathrm{eV}$ ).

\begin{tabular}{|c|c|c|c|c|c|}
\hline & \multicolumn{4}{|c|}{ Ion pair } & \\
\hline & $\mathrm{Cl}-\mathrm{C}_{2}$ & $\mathrm{Cl}-\mathrm{N}_{1}$ & $\mathrm{Cl}-\mathrm{N}_{2}$ & $\mathrm{Cl}-\mathrm{C}_{1}$ & \\
\hline $\mathrm{FF}$ & 3.04 & 3.58 & 4.26 & 3.30 & \\
\hline LDA & 2.95 & 3.54 & 4.16 & 3.32 & \\
\hline PBE & 2.99 & 3.65 & 4.18 & 3.48 & \\
\hline DRSLL & 3.04 & 3.74 & 4.21 & 3.61 & \\
\hline$\kappa=1$ & 3.03 & 3.71 & 4.21 & 3.56 & \\
\hline \multirow[t]{3}{*}{ MP2 } & 3.07 & 3.72 & 4.24 & 3.53 & \\
\hline & \multicolumn{4}{|c|}{ Dimer of ion pairs } & \\
\hline & $\mathrm{Cl}-\mathrm{C}_{5}$ & $\mathrm{Cl}-\mathrm{C}_{1}$ & $\mathrm{C}_{5}-\mathrm{C}_{5}$ & $\mathrm{Cl}-\mathrm{Cl}$ & $\Delta E$ \\
\hline $\mathrm{FF}$ & 3.15 & 3.31 & 3.47 & 6.64 & 1.77 \\
\hline LDA & 3.20 & 3.31 & 3.41 & 6.80 & 1.00 \\
\hline PBE & 3.30 & 3.43 & 3.71 & 6.85 & 0.74 \\
\hline DRSLL & 3.37 & 3.49 & 3.84 & 6.94 & 0.64 \\
\hline$\kappa=1$ & 3.35 & 3.45 & 3.70 & 6.97 & 0.70 \\
\hline MP2 & 3.34 & 3.51 & 3.63 & 6.99 & 0.92 \\
\hline
\end{tabular}

Starting from the configurations with the in-plane chlorine, we have optimized the geometry of the $[\mathrm{mmim}][\mathrm{Cl}]$ ionic pair and dimer using the four different functionals, and also at the MP2/6-31G* quantum-chemical level. In Table XI, we report some selected distances for the ionic pair and the dimer. A first observation is that PBE and VDW geometries are in better agreement with MP2 results than LDA and the FF. Second, dispersion interactions push the $\mathrm{Cl}^{-}$anion slightly away from the imidazolium ring, which is reflected in the geometry of the dimer. In summary, the inclusion of dispersion interactions appears to correct the size of the clusters in a similar way as the volumes of crystalline phases, although the PBE functional does a very respectable job compared to MP2. The all-atom FF of Youngs et al. ${ }^{6}$ is in good agreement with LDA results, generally underestimating distances and overbinding. This can be explained by the observation that, even if this FF was fitted to GGA-PBE simulations in the liquid phase using a force-matching method, no information about the equilibrium density was introduced. As a result, the density of the liquid corresponding to the FF turned out to be higher than the PBE density, and quite close to the LDA one. Dimer binding energies are all in the same region except for the LDA which, as usual, overbinds. The MP2/6-31G* value presents only a slight improvement to the LDA value, but coupled cluster calculations at the $\operatorname{CCSD}(\mathrm{T})$ level with the same basis set and geometry produce a smaller binding energy of $0.80 \mathrm{eV}$, approaching the PBE and VDW values.

Ionization energies range from $5.3 \mathrm{eV} /$ pair in the LDA to $4.9 \mathrm{eV} /$ pair in the VDW functionals. These values are consistent with the fact that the chloride anion produces stronger electrostatic bonds than the weaker $\mathrm{PF}_{6}^{-}$. Interestingly, this is not reflected in cohesive energies, at least within all the DFT approaches studied here (see the [mmim]-based compounds in Table VII), meaning that there is an interplay between geometry (anion-cation distance), and dispersion and electrostatic interactions. Nevertheless, when comparing 
energy densities, the chlorides are shown to store energy slightly more efficiently than the hexafluorophosphates.

\section{CONCLUSIONS AND DISCUSSION}

In this work, we have tested the performance of a nonempirical density functional that includes semilocal (GGA) and fully non-local van der Waals correlations on the same footing. ${ }^{11}$ Thanks to an efficient algorithm to calculate the double integral required by the non-local correlation kernel, ${ }^{12}$ we were able to conduct full geometry optimizations for supercells containing up to 224 atoms on a single processor, with a computational cost only marginally larger than a regular GGA calculation.

The results presented here for a family of imidazoliumbased ionic crystals are very encouraging. We observed a consistent improvement in equilibrium volumes with respect to both LDA and GGA-PBE for the whole family. The average error is about $7.5 \%$, which is in line with results obtained for the rare gas and benzene dimers as well as for solid argon. Binding energies could not be compared to higher-level calculations, but the effect of dispersion interactions is expected to be mitigated with respect to pure VDW complexes due to the sizable electrostatic contribution. Intra-molecular geometries were not significantly modified, as expected for covalent bonds, but there was a spectacular improvement of intermolecular geometries, especially for $[\mathrm{ddmim}]\left[\mathrm{PF}_{6}\right]$, which has the largest cation and thus the most substantial dispersion contribution. Here the average error with respect to experimental data decreased from 5\% (LDA) and 6\% (GGA-PBE) to only $2 \%$. This level of accuracy is similar to that obtained with an empirical VDW functional which, however, required a significant effort in terms of parametrization, and used a fair amount of experimental data. ${ }^{9}$

A modified VDW functional where the GGA exchange term was tuned through the $\kappa$ parameter to reproduce the energetics of water clusters ${ }^{15}$ turned out to produce better results in terms of geometries, volumes, and lattice parameters, while increasing binding energies. This is not surprising, seeing as the parameter $\kappa=1$ was obtained by optimizing the exchange functional with respect to the S22 dataset of weakly interacting dimers of biological relevance, for which the original DRSLL functional turns out to underbind. ${ }^{15}$ Therefore, while this optimization strategy helps, e.g., with the energetics of water clusters, it is unclear whether it is an effective, general road to improve over the DRSLL functional, or not. In fact, overbinding was already reported for the methane dimer, which is closer to systems studied here. ${ }^{15}$ An alternative proposed in Ref. 15 is to replace the PBE with Becke's exchange functional, ${ }^{52}$ and optimize the parameters therein. These, and other modifications to the exchange functional proposed in the past year ${ }^{53,54}$ claim a higher accuracy than the original DRSLL. We have not explored these approaches in the present work, although they appear to be promising avenues. Nevertheless, it has to be recognized that GGA semi-local exchange functionals may be insufficient. The non-locality of exchange interactions can be introduced, e.g., through hybrid HartreeFock/DFT approaches. It would be very interesting to study the interplay between (non-local) exact exchange and the non- local VDW correlation, as recently implemented by Vydrov and van Voorhis. ${ }^{55}$ Even if both non-local exchange and correlation tend to reduce bond lengths and increase binding energies, they do so via different mechanisms and at different length scales. Therefore, it would not be unlikely that, for bond lengths in the VDW region, i.e., 4-5 $\AA$, the non-local exchange turned out to reduce binding energies.

Through the present calculations we were able to shed some light into the controversy regarding the crystal structure of $[\mathrm{bmim}][\mathrm{Cl}]$. In contrast with both, LDA/GGA and force field calculations, results obtained with the DRSLL and $\kappa=1$ functionals produce much smaller energy differences between the two experimentally observed polymorphs, i.e., smaller than $0.01 \mathrm{eV}$ per ionic pair. These results are consistent with the observation of both the monoclinic and orthorhombic structures and with the results of Jayaraman and Maginn, ${ }^{46}$ and they do not rule out the possibility of a temperature-driven phase transition between them.

We have also studied the structure and energetics of ionic pairs and dimers, which is an important issue for the formation of clusters and nanodroplets. Structural properties, in particular inter-molecular distances, are severely affected by the choice of functional in the same way as volumes of crystalline phases, with the disadvantage that one cannot enforce it by choosing an appropriate box. A widespread approach is to use force fields fitted to accurate quantum-chemical calculations in ionic pairs, but it always remains the doubt of how transferable these force fields are to condensed phases. For [bmim][Tf], the present calculations show that the two VDW functionals and also PBE produce geometries in excellent agreement with MP2/6-31G* calculations. The force field of Ref. 3 tends to overbind but overall does a reasonable job. The LDA, as usual, produces very small distances. Binding energies for the dimer are comparable at the various levels. This, however, is likely to be a consequence of error cancellations.

The results obtained for [mmim] $[\mathrm{Cl}]$ pair and dimer exhibit similar trends, but the agreement with MP2/6-31G* geometries and energies is even better than for [bmim][Tf]. Here the VDW functionals perform better than PBE. The force field used for $[\mathrm{mmim}][\mathrm{Cl}]$ was obtained by a force-matching fitting procedure to GGA-PBE simulations in the liquid phase, and turned out to produce a volume smaller than experiment. Therefore, it is not a good reference for comparison. Nevertheless, we showed that the cluster structures produced by this force field are retained by all functionals.

Summarizing, van der Waals (dispersion) interactions are now available in density functional theory, at a very modest computational cost, and they improve equilibrium volumes and internal geometries of room-temperature ionic liquids to an excellent extent without compromising the good performance of semi-local functionals for chemical bonds.

\section{ACKNOWLEDGMENTS}

We are thankful to Mario Del Pópolo for facilitating some of the data and structures, and for useful ideas and discussions. This work is inscribed within the research programme of QUILL at Queen's University Belfast, funded through 
EPSRC Grant No. EP/D029538/1, and was initiated in the Department of Earth Sciences at the University of Cambridge during J.K.'s sabbatical leave. J.M.S. acknowledges support of project FIS2009-12721 by Spain's MCI. C.P. acknowledges funding from EU-FP7-NMP Grant No. 229205.

${ }^{1}$ Ionic Liquids: Industrial Applications for Green Chemistry, edited by R. D. Rogers and K. R. Seddon (American Chemical Society, Washington, DC, 2002).

${ }^{2}$ R. M. Lynden-Bell, M. G. Del Pópolo, T. G. A. Youngs, J. Kohanoff, C. G. Hanke, J. B. Harper, and C. Pinilla, Acc. Chem. Res. 40, 1138 (2007).

${ }^{3}$ J. N. Canongia Lopes, J. Deschamps, and A. A. H. Padua, J. Phys. Chem. B 108, 2038 (2004); 108, 11250 (2004); J. N. Canongia Lopes and A. A. H. Padua, ibid. 108, 16893 (2004).

${ }^{4}$ Z. P. Liu, S. P. Huang, and W. C. Wang, J. Phys. Chem. B 108, 12978 (2004).

${ }^{5}$ M. G. Del Pópolo, R. M. Lynden-Bell, and J. Kohanoff, J. Phys. Chem. B 109, 5895 (2005).

${ }^{6}$ T. G. A. Youngs, M. G. Del Pópolo, and J. Kohanoff, J. Phys. Chem. B 110, 5697 (2006).

${ }^{7}$ C. Hardacre, J. D. Holbrey, S. E. J. McMath, D. T. Bowron, and A. K. Soper, J. Chem. Phys. 118, 273 (2003); C. Hardacre, S. E. J. McMath, M. Nieuwenhuyzen, D. T. Bowron, and A. K. Soper, J. Phys.: Condens. Matter 15, S159 (2003).

${ }^{8}$ J. P. Perdew, K. Burke, and M. Ernzerhof, Phys. Rev. Lett. 77, 3865 (1996); 78, 1396(E) (1997).

${ }^{9}$ M. G. Del Pópolo, C. Pinilla, and P. Ballone, J. Chem. Phys. 126, 144705 (2007).

${ }^{10}$ M. Elstner, P. Hobza, S. Suhai, and E. Kaxiras, J. Chem. Phys. 114, 5149 (2001); X. Wu, M. C. Vargas, S. Nayak, V. Lotrich, and G. Scoles, ibid. 115, 8748 (2001); Q. Wu and W. Yang, ibid. 116, 515 (2002); S. Grimme, J. Comput. Chem. 25, 1463 (2004).

${ }^{11}$ M. Dion, H. Rydberg, E. Schröder, D. C. Langreth, and B. I. Lundqvist, Phys. Rev. Lett. 92, 246401 (2004); 95, 109902(E) (2005).

${ }^{12}$ G. Román-Pérez and J. M. Soler, Phys. Rev. Lett. 103, 096102 (2009).

${ }^{13}$ J. M. Soler, E. Artacho, J. D. Gale, A. García, J. Junquera, P. Ordejón, and D. Sánchez-Portal, J. Phys.: Condens. Matter 14, 2745 (2002).

${ }^{14}$ P. Ballone, C. Pinilla, J. Kohanoff, and M. G. Del Pópolo, J. Phys. Chem. B 111, 4938 (2007).

${ }^{15}$ J. Klimes, D. Bowler, and A. Michaelides, J. Phys.: Condens. Matter 22, 074203 (2010).

${ }^{16} \mathrm{See}$, for example, Lennard-Jones parameters in the OPLS force-field: W. L. Jorgensen, D. S. Maxwell, and J. Tirado-Rives, J. Am. Chem. Soc. 118, 11225 (1996).

${ }^{17}$ Y. Zhang and W. Yang, Phys. Rev. Lett. 80, 890 (1998). This functional is a simple re-parameterization of PBE, with $\kappa=1.245$ instead of 0.804 .

${ }^{18}$ See, for instance, W. Kohn, Y. Meir, and D. E. Makarov, Phys. Rev. Lett. 80, 4153 (1998); H. Rydberg, B. I. Lundqvist, D. C. Langreth, and M. Dion, Phys. Rev. B 62, 6997 (2000); H. Rydberg, M. Dion, N. Jacobsen, E. Schrder, P. Hyldgaard, S. I. Simak, D. C. Langreth, and B. I. Lundqvist, Phys. Rev. Lett. 91, 126402 (2003); T. A. Wesolowski and F. Tran, J. Chem. Phys. 118, 2072 (2003); E. R. Johnson and A. D. Becke, ibid. 124, 174104 (2006); A. Tkatchenko and M. Scheffler, Phys. Rev. Lett. 102, 073005 (2009).

${ }^{19}$ D. C. Langreth, B. I. Lundqvist, S. D. Chakarova-Käck, V. R. Cooper, M. Dion, P. Hyldgaard, A. Kelkkanen, J. Kleis, L. Kong, S. Li, P. G. Moses, E. Murray, A. Puzder, H. Rydberg, E. Schröder, and T. Thonhauser, J. Phys.: Condens. Matter 21, 084203 (2009).

${ }^{20}$ J. Klimes, D. R. Bowler, and A. Michaelides, Phys. Rev. B 83, 195131 (2011).

${ }^{21}$ J. Hooper, V. R. Cooper, T. Thonhauser, N. A. Romero, F. Zerilli, and D. C. Langreth, Chem. Phys. Chem. 9, 891 (2008).

${ }^{22}$ J. Kleis, B. I. Lundqvist, D. C. Langreth, and E. Schröder, Phys. Rev. B 76, 100201 (2007)

${ }^{23}$ T. Thonhauser, V. R. Cooper, S. Li, A. Puzder, P. Hyldgaard, and D. C. Langreth, Phys. Rev. B 76, 125112 (2007).

${ }^{24}$ J. P. Perdew, A. Ruzsinszky, G. I. Csonka, O. A. Vydrov, G. E. Scuseria, L. A. Constantin, X. Zhou, and K. Burke, Phys. Rev. Lett. 100, 136406 (2008)

${ }^{25}$ E. H. Lieb and S. Oxford, Int. J. Quantum Chem. 19, 427 (1981).

${ }^{26}$ J. A. Alonso and L. A. Girifalco, Solid State Commun. 24, 135 (1977); Phys. Rev. B 17, 3735 (1978); O. Gunnarsson, M. Jonson, and
B. Lundqvist, Solid State Commun. 24, 765 (1977). See also D. J. Singh, Phys. Rev. B 48, 14099 (1993).

${ }^{27}$ N. Troullier and J. L. Martins, Phys. Rev. B 43, 1993 (1991).

${ }^{28}$ E. Artacho, D. Sánchez-Portal, P. Ordejón, A. García, and J. M. Soler, Phys. Status Solidi B 215, 809 (1999).

${ }^{29}$ J. Junquera, O. Paz, D. Sánchez-Portal, and E. Artacho, Phys. Rev. B 64, 235111 (2001)

${ }^{30}$ E. Artacho, E. Anglada, O. Diéguez, J. D. Gale, A. García, J. Junquera, R. M. Martin, P. Ordejón, J. M. Pruneda, D. Sánchez-Portal, and J. M. Soler, J. Phys.: Condens. Matter 20, 064208 (2008).

${ }^{31}$ J. Moreno and J. M. Soler, Phys. Rev. B 45, 13891 (1992).

${ }^{32}$ I. Scivetti, N. Gidopoulos, and J. Kohanoff, Phys. Rev. B 78, 224108 (2008).

${ }^{33}$ M. Leslie and N. J. Gillan, J. Phys. C: Solid State Phys. 18, 973 (1985); see also G. Makov and M. Payne, Phys. Rev. B 51, 4014 (1995).

${ }^{34}$ Experimental data for the hexafluorophosphate crystals have been taken from the following references: $[\mathrm{mmim}]\left[\mathrm{PF}_{6}\right] \mathrm{J}$. D. Holbrey, W. M. Reichert, M. Nieuwenhuyzen, O. Sheppard, C. Hardacre, and R. D. Rogers, Chem. Commun. (Cambridge) 2003, 476 (2003); [emim] $\left[\mathrm{PF}_{6}\right]$ B. L. Barker, G. G. Stanley, and F. R. Fronczek, Cambridge Crystallographic Data Centre, Deposition Number: 228745 (2004); [bmim][PF 6$]$ S. M. Dibrov and J. K. Kochi, Acta Crystallogr., Sect. C: Cryst. Struct. Commun. C62, o19 (2006); [ddmim] $\left[\mathrm{PF}_{6}\right]$ C. M. Gordon, J. D. Holbrey, A. Kennedy, and K. R. Seddon, J. Mater. Chem. 8, 2627 (1998).

${ }^{35}$ Experimental data for the chloride crystals has been taken from the following references: $[\mathrm{mmim}][\mathrm{Cl}]$ A. J. Arduengo III, H. V. Rasika Dias, R. L. Harlow, and M. Kline, J. Am. Chem. Soc. 114, 5530 (1992); [bmim][Cl] J. D. Holbrey, W. M. Reichert, N. Nieuwenhuyzen, S. Johnston, K. R. Seddon, and R. D. Rogers, Chem. Commun. (Cambridge) 2003, 1636 (2003).

${ }^{36}$ B. Montanari and R. O. Jones, Chem. Phys. Lett. 272, 347 (1997); B. Montanari, P. Ballone, and R. O. Jones, J. Chem. Phys. 108, 6947 (1998).

${ }^{37}$ Notice that the agreement between the $\kappa=1$ volumes and the ones measured at finite temperature (last column in Table II) is even closer than the $T=0 \mathrm{~K}$ extrapolations. This improved agreement, however, is fortuitous and most likely due to limitations of the functional.

${ }^{38}$ A. J. Arduengo III, H. V. Rasika Dias, R. L. Harlow, and M. Kline, J. Am Chem. Soc. 114, 5530 (1992)

${ }^{39}$ J. D. Holbrey, W. M. Reichert, M. Nieuwenhuyzen, S. Johnston, K. R. Seddon, and R. D. Rogers, Chem. Comm. 2003, 1636 (2003).

${ }^{40}$ J. D. Holbrey, W. M. Reichert, M. Nieuwenhuyzen, O. Sheppard, C. Hardacre, and R. D. Rogers, Chem. Comm. 2003, 476 (2003).

${ }^{41}$ B. L. Barker, G. G. Stanley, and F. R. Fronczek, Cambridge Crystallographic Data Centre, Deposition Number: 228745 (2004).

${ }^{42}$ S. M. Dibrov and K. Kochi, Acta Crystallogr., Sect. C: Cryst. Struct. Commun. C62, o19 (2006).

${ }^{43}$ C. M. Gordon, J. D. Holbrey, A. Kennedy, and K. R. Seddon, J. Mater. Chem. 8, 2627 (1998).

${ }^{44}$ S. Hayashi, R. Ozawa, and H.-O. Hamaguchi, Chem. Lett. 32, 498 (2003); H.-O. Hamaguchi and R. Ozawa, Adv. Chem. Phys. 135, 85 (2005).

${ }^{45}$ The original LDA and PBE cohesive energies calculated in (Ref. 9) were somewhat smaller due to BSSE effects, and which were corrected in the present calculations.

${ }^{46}$ S. Jayaraman and E. J. Maginn, J. Chem. Phys. 127, 214504 (2007).

${ }^{47}$ C. Pinilla, Ph.D. dissertation, Queen's University Belfast, 2007.

${ }^{48}$ M. J. Earle, J. M. S. S. Esperança, M. A. Gilea, J. N. Canongia Lopes, L. P. N. Rebelo, J. W. Magee, K. R. Seddon, and J. A. Widegren, Nature (London) 439, 831 (2006); L. P. N. Rebelo, J. N. Canongia Lopes, J. M. S. S. Esperança, and E. J. Filipe, J. Phys. Chem. B 109, 6040 (2005).

${ }^{49}$ M. W. Schmidt, K. K. Baldridge, J. A. Boatz, S. T. Elbert, M. S. Gordon, J. H. Jensen, S. Koseki, N. Matsunaga, K. A. Nguyen, S. J. Su, T. L. Windus, M. Dupuis, and J. A. Montgomery, J. Comput. Chem. 14, 1347 (1993); M. S. Gordon and M. W. Schmidt, in Theory and Applications of Computational Chemistry, the First Forty Years, edited by C. E. Dykstra, G. Frenking, K. S. Kim, and G. E. Scuseria (Elsevier, Amsterdam, 2005), Ch. 41, pp. 1167-1189.

${ }^{50}$ O. Borodin, J. Phys. Chem. B 113, 11463 (2009).

${ }^{51}$ S. L. Price, C. G. Hanke, and R. M. Lynden-Bell, Mol. Phys. 99, 801 (2001).

${ }^{52}$ A. D. Becke, Phys. Rev. A 38, 3098 (1988).

${ }^{53}$ V. R. Cooper, Phys. Rev. B 81, 161104(R) (2010).

${ }^{54}$ K. Lee, E. D. Murray, L. Kong, B. I. Lundqvist, and D. C. Langreth, Phys. Rev. B 82, 081101(R) (2010).

${ }^{55}$ O. Vydrov and T. Van Voorhis, Phys. Rev. Lett. 103, 063004 (2009). 